\title{
Multiscale modelling of auxin transport in the plant-root elongation zone
}

\author{
L. R. Band • J. R. King
}

Received: 17 September 2010 / Revised: 17 August 2011 / Published online: 20 October 2011 C The Author(s) 2011. This article is published with open access at Springerlink.com

\begin{abstract}
In the root elongation zone of a plant, the hormone auxin moves in a polar manner due to active transport facilitated by spatially distributed influx and efflux carriers present on the cell membranes. To understand how the cell-scale active transport and passive diffusion combine to produce the effective tissue-scale flux, we apply asymptotic methods to a cell-based model of auxin transport to derive systematically a continuum description from the spatially discrete one. Using biologically relevant parameter values, we show how the carriers drive the dominant tissue-scale auxin flux and we predict how the overall auxin dynamics are affected by perturbations to these carriers, for example, in knockout mutants. The analysis shows how the dominant behaviour depends on the cells' lengths, and enables us to assess the relative importance of the diffusive auxin flux through the cell wall. Other distinguished limits are also identified and their potential roles discussed. As well as providing insight into auxin transport, the study illustrates the use of multiscale (cell to tissue) methods in deriving simplified models that retain the essential biology and provide understanding of the underlying dynamics.
\end{abstract}

Keywords Multiscale - Asymptotic analysis · Plant root · Auxin · Hormone transport · Polar transport

Mathematics Subject Classification (2000) $\quad 34 \mathrm{E} 13 \cdot 92 \mathrm{C} 80$

\footnotetext{
L. R. Band $(\varangle) \cdot$ J. R. King

Centre for Plant Integrative Biology, University of Nottingham,

Sutton Bonington, Nottingham LE12 5RD, UK

e-mail: leah.band@cpib.ac.uk

J. R. King

School of Mathematical Sciences, University of Nottingham,

University Park, Nottingham NG7 2RD, UK
} 


\section{Introduction}

To understand biological phenomena, we must consider processes that occur over multiple spatial and/or temporal scales, determining how behaviour at, say, the tissue scale emerges as a result of the cell-scale dynamics. Such behaviour can be understood using multiscale asymptotic methods which enable us to derive simplified descriptions of the tissue-scale dynamics, identifying the key contributions of the cell-scale processes. In this paper, we use asymptotic methods to gain understanding of the transport of the hormone auxin through plant tissue. Auxin plays an important role in plant growth and development (Benjamins and Scheres 2008; Friml 2003; Tanaka et al. 2006; Vieten et al. 2007), and an asymptotic analysis is particularly applicable since plant tissue has a regular structure and we can distinguish the roles of the several cell-scale components of the auxin flux. Thus, the analysis presented provides insight into the underlying dynamics and complements the many computational cell-scale auxin-transport models that are currently in development [see Berleth et al. (2007); Jönsson and Krupinski (2010); Kramer (2008); Kramer et al. (2008); Krupinski and Jönsson (2010) and Smith and Bayer (2009) for recent reviews].

Our analysis focusses on the polar auxin transport in the root elongation zone of the model species Arabidopsis thaliana (Fig. 1). In the root, auxin regulates the gravitropic response (Marchant et al. 1999; Ottenschlager et al. 2003), maintains the root apical meristem (Jiang and Feldman 2005) and promotes the initiation of lateral roots (Benková et al. 2003; Casimiro et al. 2003, 2001; De Smet et al. 2007). Therefore, understanding the distribution and transport of auxin within root tissue could help explain key processes in root development. Auxin flows towards the root apex (i.e. in the rootward direction) in the centre of the root and in an upwards (shootward) direction though the root's outer layers. The flux through the root's outer layers is important in producing a gravitropic response (Rashotte et al. 2000; Swarup et al. 2005): if the root is not orientated downwards (in the direction of gravity) the columella cells, located at the root tip, create a lateral auxin gradient (Ottenschlager et al. 2003); due to the upward flux though the root's outer layers, the elongation-zone cells on the underside of the root receive more auxin than those on the upper side, which causes them to reduce their elongation rate; within minutes, this process causes the root to bend and reorientate in the direction of gravity (Friml 2003; Swarup et al. 2005; Tanaka et al. 2006).

Away from the tip, the primary Arabidopsis thaliana root consists of three concentric single-cell layers, the epidermis, cortex and endodermis, which in turn surround the pericycle cells and vasculature (see Fig. 1). As the root grows, cells are produced close to the root tip and pass through the elongation zone, increasing in length by approximately 20-fold (Swarup et al. 2007). Each cell consists of a cytoplasm, containing the vacuole and other cytoplasmic organelles, enclosed by a plasma membrane that is embedded in a connective tissue layer called the apoplast (or cell wall). Auxin moves through the tissue by several mechanisms: it diffuses through the cell cytoplasm and apoplast, and it moves between the cytoplasms and apoplast by crossing the plasma membrane. Within the tissue, auxin is present in both a protonated form, $I A A H$, and an anionic form, $I A A^{-}$; protonated auxin permeates cell membranes by passive diffusion, whereas the anionic form passes through cell membranes by active transport mediated by influx and efflux carriers (Kramer and Bennett 2006). There 
Fig. 1 The Arabidopsis thaliana root has a layered structure. The cells divide in the meristem, and then increase in length approximately 20 -fold as they move through the elongation zone [Figure adapted from Péret et al. (2009)]

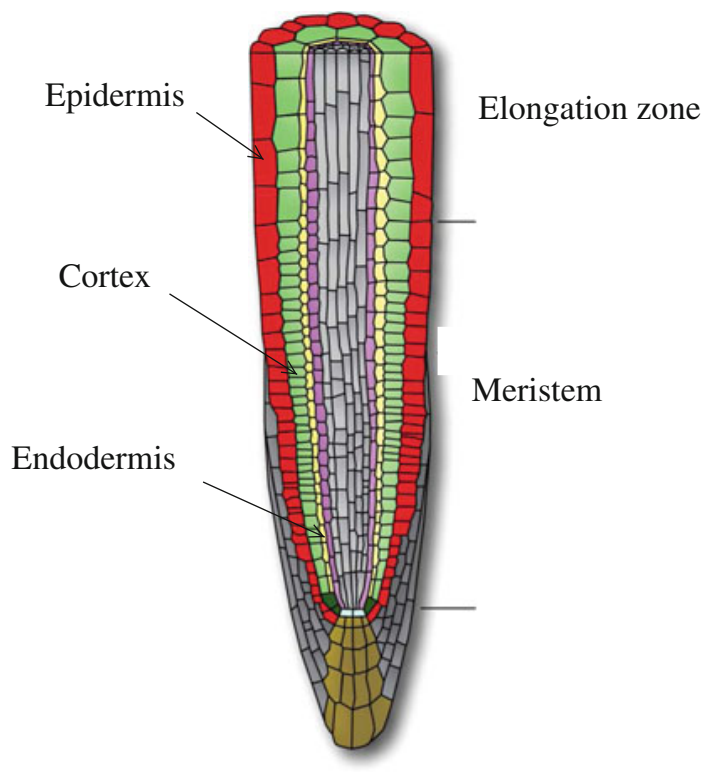

are significant amounts of both anionic and protonated auxin present in the acidic environment of the apoplast, so both passive diffusion and active carrier-mediated transport contribute to the auxin flux into the cytoplasms. When auxin enters a cell cytoplasm, where the $\mathrm{pH}$ is higher than in the apoplast, the majority of the protonated auxin rapidly dissociates resulting in predominantly anionic auxin that can leave the cytoplasm only via the efflux carriers (Kramer and Bennett 2006).

Key efflux and influx carriers are the PIN and AUX1 protein families, respectively. Efflux carriers are located asymmetrically (see Fig. 2) and can generate a net flux through the tissue. Considering the outer tissue layers in the root elongation zone, the epidermal cell membranes contain PIN1 and PIN2 carriers on their shootward face (upwards), whereas the endodermal cell membranes contain PIN1 carriers only, on their rootward face (downwards) (Blilou et al. 2005). On the cortical cell membranes, the location of the PIN1 and PIN2 carriers is currently unclear, with some sources reporting a rootward location (Blilou et al. 2005) and others a shootward one (Peer et al. 2004). There are also spatial variations in the AUX1 influx carriers, these being present on all sides of the epidermal cell membranes, but not on the cortical and endodermal cell membranes (Swarup et al. 2005). With this spatial distribution, the carriers create a shootward auxin flux in the outer layers of the elongation zone that balances the rootward flux through the root centre (Blilou et al. 2005; Friml et al. 2002). In Arabidoposis thaliana, various mutants demonstrate the importance of the carriers; for example, both auxl and pin2 mutants (which have dysfunctional AUX1 carriers and PIN2 carriers, respectively) have disrupted auxin transport and are agravitropic (Rashotte et al. 2000; Swarup et al. 2005). Such mutations will be explored within our model framework below.

Various cell-based models of auxin transport have been developed and have provided insight into auxin's role in the gravitropic response (Grieneisen et al. 2007; 


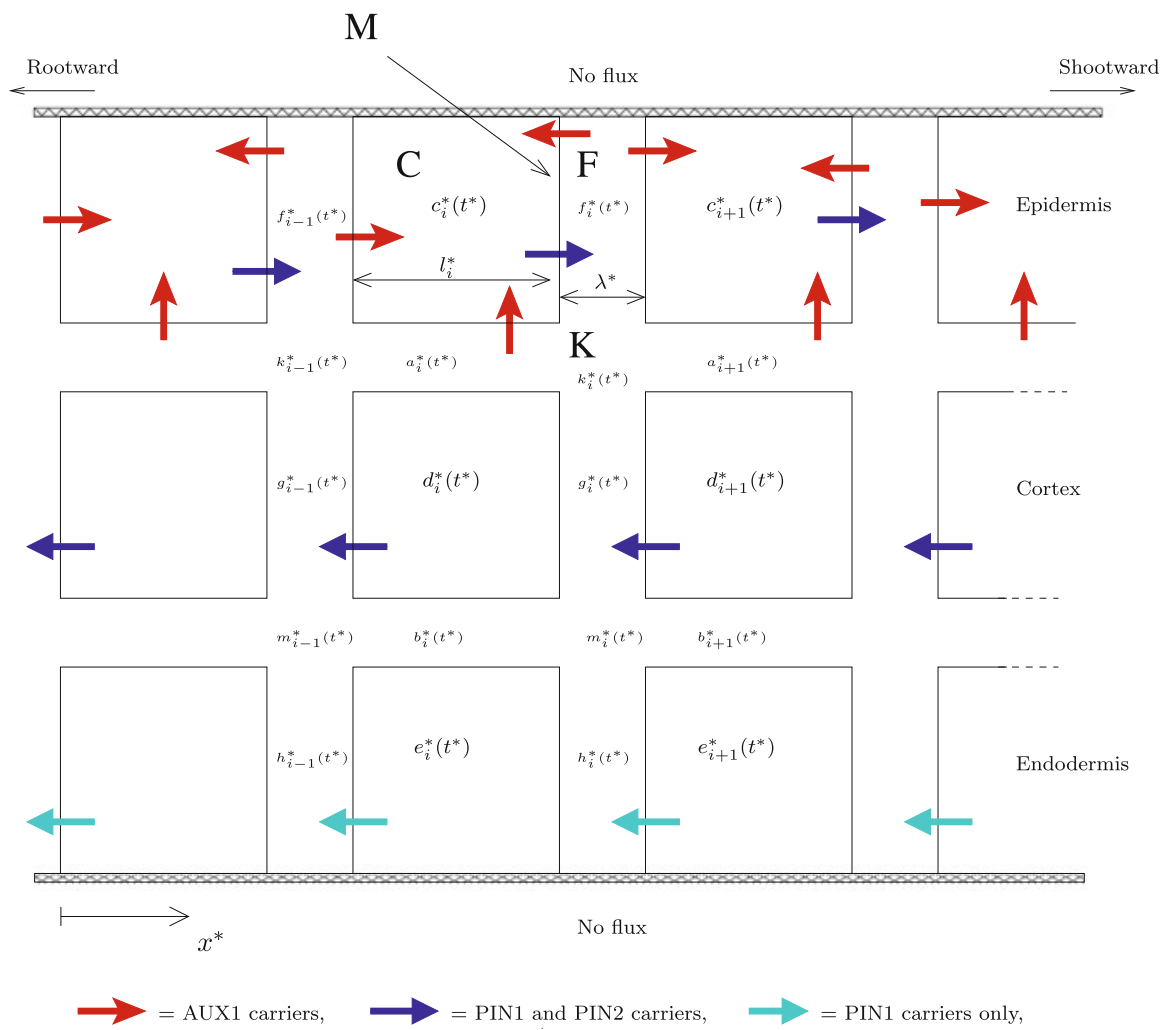

Fig. 2 We consider a two-dimensional model which consists of three rows of cells representing the epidermal, cortical and endodermal layers. Each epidermal cell expresses AUX1 influx carriers on all sides of its membrane and PIN1 and PIN2 efflux carriers on the shootward side. The cortical and endodermal cells do not express influx carriers, but express efflux carriers on their rootward side, with the cortical cells expressing both PIN1 and PIN2 and the endodermal cells only PIN1. The labels C, F, K, and M are provided to clarify the discussion in Sect. 2

Swarup et al. 2005), vein formation (Feugier et al. 2005; Feugier and Iwasa 2006; Merks et al. 2007; Mitchison 1980a; Mitchison et al. 1981; Rolland-Lagan and Prusinkiewicz 2005) and organ initiation (de Reuille et al. 2006; Heisler and Jönsson 2006; Jönsson et al. 2006; Laskowski et al. 2008; Stoma et al. 2008; Newell et al. 2008; Perrine-Walker et al. 2010; Smith et al. 2006; Stoma et al. 2008), for example. Early studies (Goldsmith et al. 1981; Martin et al. 1990; Mitchison 1980b) considered only a single line of cells, with auxin diffusion within the cytoplasms and with passive and carrier-mediated transport across the cell membranes; these showed how the efflux carriers increase the tissue-scale auxin flux. More recent studies have considered more complex tissue geometries and have incorporated apoplastic diffusion (Heisler and Jönsson 2006; Jönsson et al. 2006; Kramer 2004; Laskowski et al. 2008; Swarup et al. 2005), saturable carrier-mediated transport (de Reuille et al. 2006; Feugier et al. 2005; Jönsson et al. 2006; Merks et al. 2007; Smith et al. 2006), cytoplasmic structure (Kramer 2004; Perrine-Walker et al. 2010), cell growth (Chavarria-Krauser et al. 
2005; Grieneisen et al. 2007; Jönsson et al. 2006; Mironova et al. 2010; Stoma et al. 2008), auxin production and degradation (Feugier and Iwasa 2006; Feugier et al. 2005; Perrine-Walker et al. 2010; Heisler and Jönsson 2006; Jönsson et al. 2006; Smith et al. 2006; Stoma et al. 2008) and dynamics of the efflux carriers (Heisler and Jönsson 2006; Jönsson et al. 2006; Kramer 2009; Merks et al. 2007; Mironova et al. 2010; Stoma et al. 2008).

In contrast to most current auxin-transport modelling, here we take an analytical approach. Using an asymptotic method, we reduce the hundreds of ordinary differential equations governing a discrete cell-based model to a few partial differential equations that describe the tissue-scale dynamics (using a continuum approximation, applicable since within each layer spatial variations in auxin concentration occur over many cell lengths). The derived equations provide insight into the underlying dynamics and show how the cell-scale parameters influence the effective velocity and diffusivity of the auxin flux. The benefits of this multiscale analytical approach have recently been highlighted by Twycross et al. (2010). Similar modelling approaches have been used to study calcium dynamics (Goel et al. 2006), pattern formation (Newell et al. 2008) and nutrient distributions (Shipley et al. 2009), for example, and the effective tissue-scale auxin transport has also been considered in Chavarría-Krauser and Ptashnyk (2009); Kramer (2002); Martin et al. (1990); Mitchison (1980a,b); Mitchison et al. (1981) and Newell et al. (2008).

Having outlined the cell-scale model in Sect. 2, we present the model analysis in Sect. 3. We describe the continuum approach, Sects. 3.1-3.3, and then demonstrate the key features of the model by considering two simple cases that provide building blocks for the subsequent more realistic analysis: in Sect. 3.4, we treat the cell membranes as impermeable, so that the cytoplasmic auxin concentrations are constant and auxin moves through the tissue by random motion within the apoplast; in Sect. 3.5, we consider the apoplast thickness to be zero and find that the efflux carriers create directed auxin flux (which manifests itself mathematically as an advective term). These simple cases enable us to make direct connections to very familiar analyses of continuum limits of discrete systems. We identify key limits of the full system in Sect. 3.6, and show how the nature of the auxin flux depends on the relative importance of the advective fluxes created by the carrier-mediated transport and the diffusive fluxes through the apoplast. The biological relevance of these asymptotic regimes is discussed in Sect. 4. In the light of experimentally estimated parameter values, we find the tissue-scale behaviour depends on the cell length. For the short cells close to the root tip, the epidermis drives the auxin flux, with the effective velocity determined by the efflux carriers in this layer, whereas where the tissue consists of longer cells further through the elongation zone, auxin distributes between the three layers on the same time scale as the tissue-scale flux so that the effective velocity depends on the distribution of carriers in each layer. In both cases, the presence of the influx carriers creates high auxin concentrations in the epidermis so that the shootward epidermal flux dominates over the rootward fluxes in the cortex and endodermis. We use the analysis to predict how the overall auxin dynamics are affected by perturbations to the carriers, for example, in knockout mutants; a summary of these key biological results can be found in Tables 5 and 6 . We also determine the importance of the apoplastic diffusion in the different cases. Our conclusions are discussed in detail in Sect. 5. 


\section{Model description}

\subsection{Model assumptions}

We base our analysis on the cell-scale model of Swarup et al. (2005), who consider auxin transport in the epidermal, cortical and endodermal layers of the root elongation zone with the distribution of efflux carriers reported by Blilou et al. (2005) (see Fig. 1). The model includes both passive and active auxin transport across the cell membranes, and auxin diffusion within the apoplast [and thus allows for the possibility that auxin travels relatively large distances through the apoplast before entering a cell cytoplasm (Kramer et al. 2007)]. In contrast to the cylinder of cells in Swarup et al. (2005), we consider for simplicity a two-dimensional configuration with each layer represented by a row of $N$ cells, the cells in each row being labelled by $i=1,2,3, \ldots, N$ [thus, all compartments are taken to be the same thickness in the third ('out-of-the-page') dimension with the fluxes in that direction being zero]. The model therefore consists of a constant array of cells and does not incorporate cell growth or cells entering and leaving the elongation zone (see Sect. 2.2 for further details). As shown in Fig. 2, we treat the cytoplasms as rectangular, with equal widths, $w^{*}$, and with lengths, $l_{i}^{*}$, that gradually increase through the elongation zone (where asterisks are used to denote dimensional quantities). A representative cytoplasm length will be denoted by $l_{T}^{*}$. We suppose that the apoplast has uniform thickness, $\lambda^{*}$, and denote the total length of the tissue by $L^{*}$. The auxin concentrations in the cytoplasms of the epidermal, cortical and endodermal cell $i$ are denoted by $c_{i}^{*}\left(t^{*}\right), d_{i}^{*}\left(t^{*}\right)$, and $e_{i}^{*}\left(t^{*}\right)$ respectively, and for convenience in model development, we divide the apoplast into distinct regions in which the auxin concentrations are denoted by $f_{i}^{*}\left(t^{*}\right), g_{i}^{*}\left(t^{*}\right), h_{i}^{*}\left(t^{*}\right), a_{i}^{*}\left(t^{*}\right), b_{i}^{*}\left(t^{*}\right), k_{i}^{*}\left(t^{*}\right)$ and $m_{i}^{*}\left(t^{*}\right)$, where $t^{*}$ denotes time (see Fig. 2 for details). We use the term cytoplasm to refer to everything enclosed within the plasma membrane and we treat each cell cytoplasm as a single compartment, i.e. we do not consider the vacuole or other cytoplasmic organelles separately. We assume that intracellular auxin transport is sufficiently fast that the cytoplasmic auxin concentrations can be treated as spatially homogeneous (as discussed in Sect. 2.2).

Within the tissue, auxin is present in both protonated and anionic forms; in each compartment, the proportion of each equilibrates rapidly and is determined by the $\mathrm{pH}$ and by the dissociation constant, $p K$, such that the proportions of protonated auxin in the apoplast and cytoplasm are given by

$$
A_{1}=\frac{1}{1+10^{p H_{a}-p K}}, \quad B_{1}=\frac{1}{1+10^{p H_{c}-p K}},
$$

respectively, where $p H_{a}$ denotes the apoplastic $\mathrm{pH}$ and $p H_{c}$ the cytoplasmic $\mathrm{pH}$. Thus, for example, in the cytoplasm of epidermal cell $i$ the concentration of protonated auxin is $A_{1} c_{i}^{*}$ and that of anionic auxin is $\left(1-A_{1}\right) c_{i}^{*}$. Auxin moves between the cell cytoplasms and the apoplast by crossing the plasma membrane. Following Swarup et al. (2005), we separate the auxin flux from a cytoplasm into the apoplast (i.e. the number of molecules passing through the membrane per unit membrane length per unit time) into distinct components, namely the passive diffusive flux, $J_{I A A H}^{*}$, the active influx 
facilitated by the AUX1 carriers, $J_{A U X 1}^{*}$, the active efflux facilitated by the PIN1 and PIN2 carriers, $J_{P I N}^{*}$, and the active efflux facilitated by the PIN1 carriers alone, $J_{P I N 1}^{*}$ (this demarcation achieves greater brevity of notation than introducing the PIN2 flux $\left.J_{P I N 2}^{*}=J_{P I N}^{*}-J_{P I N 1}^{*}\right)$. With no carriers, the membrane is impermeable to anionic auxin and the passive diffusive flux, $J_{I A A H}^{*}$, is driven by the concentration difference in protonated auxin (Swarup et al. 2005). Thus, considering the cell membrane face labelled $M$ on Fig. 2, for example, the passive flux per unit length from the cytoplasm labelled $\mathrm{C}$ into the apoplast region labelled $\mathrm{F}$ is given by

$$
J_{I A A H}^{*}=P_{I A A H}^{*}\left(B_{1} c_{i}^{*}-A_{1} f_{i}^{*}\right)
$$

where $P_{I A A H}^{*}$ is the passive membrane permeability (i.e. the number of molecules crossing the membrane per unit membrane length per unit concentration difference per unit time). In contrast, the influx and efflux carriers actively transport anionic auxin by exploiting the electrochemical gradient across the cell membrane. For simplicity, we assume that the carriers are not saturable and do not consider any carrier dynamics (i.e. we neglect regulation of the PIN carriers). As in previous studies [Kramer et al. (2008); Krupinski and Jönsson (2010) and references therein], we model the carriermediated flux using Goldman-Hodgkin-Katz theory [see Keener and Sneyd (2004) for further details]. As shown in Appendix A, this results in the carrier-mediated fluxes from the cytoplasm labelled $\mathrm{C}$ into the apoplast region labelled $\mathrm{F}$ being of the form

$$
\begin{aligned}
J_{A U X 1}^{*} & =-P_{A U X 1}^{*}\left(A_{2} f_{i}^{*}-B_{2} c_{i}^{*}\right), \\
J_{P I N}^{*} & =P_{P I N}^{*}\left(B_{3} c_{i}^{*}-A_{3} f_{i}^{*}\right),
\end{aligned}
$$

where constants $A_{2}, A_{3}, B_{2}$ and $B_{3}$ are given by

$$
\begin{aligned}
& A_{2}=q(\tilde{\phi})\left(1-A_{1}\right), \quad A_{3}=q(-\tilde{\phi})\left(1-A_{1}\right), \\
& B_{2}=q(-\tilde{\phi})\left(1-B_{1}\right), \quad B_{3}=q(\tilde{\phi})\left(1-B_{1}\right), \\
& \text { where } q(\tilde{\phi})=\tilde{\phi} /\left(e^{\tilde{\phi}}-1\right) \text { and } \tilde{\phi}=F_{D} V^{*} / R T^{*} .
\end{aligned}
$$

Here $F_{D}$ is Faraday's constant, $R$ is the gas constant, $T^{*}$ is the temperature and $V^{*}$ is the potential difference across the membrane. For the endodermal cells, the active efflux due to PIN1 carriers alone is given by $(2.3 \mathrm{~b})$ with $J_{P I N}^{*}$ and $P_{P I N}^{*}$ replaced by $J_{P I N 1}^{*}$ and $P_{P I N 1}^{*}$. We note that under the above assumptions, the carrier-mediated fluxes have passive components [see Appendix A], which result in the influx carriers creating a small efflux, and the efflux carriers creating a small influx.

We can now formulate the total flux per unit length through cell membrane face $\mathrm{M}$ as the sum of the three flux components, $J_{I A A H}^{*}+J_{P I N}^{*}+J_{A U X 1}^{*},(2.2,2.3)$. Similarly, considering the flux contributions that are appropriate for each face of each cell membrane of the cytoplasms labelled $i$, the various auxin fluxes per unit length are given by

$$
\begin{aligned}
J_{c f i}^{*}= & \left(B_{1} P_{I A A H}^{*}+B_{2} P_{A U X 1}^{*}+B_{3} P_{P I N}^{*}\right) c_{i}^{*} \\
& -\left(A_{1} P_{I A A H}^{*}+A_{2} P_{A U X 1}^{*}+A_{3} P_{P I N}^{*}\right) f_{i}^{*}, \\
J_{f c i}^{*}= & \left(A_{1} P_{I A A H}^{*}+A_{2} P_{A U X 1}^{*}\right) f_{i-1}^{*}-\left(B_{1} P_{I A A H}^{*}+B_{2} P_{A U X 1}^{*}\right) c_{i}^{*},
\end{aligned}
$$




$$
\begin{aligned}
J_{c a i}^{*} & =\left(B_{1} P_{I A A H}^{*}+B_{2} P_{A U X 1}^{*}\right) c_{i}^{*}-\left(A_{1} P_{I A A H}^{*}+A_{2} P_{A U X 1}^{*}\right) a_{i}^{*}, \\
J_{d g i}^{*} & =B_{1} P_{I A A H}^{*} d_{i}^{*}-A_{1} P_{I A A H}^{*} g_{i}^{*}, \\
J_{g d i}^{*} & =\left(A_{1} P_{I A A H}^{*}+A_{3} P_{P I N}^{*}\right) g_{i-1}^{*}-\left(B_{1} P_{I A A H}^{*}+B_{3} P_{P I N}^{*}\right) d_{i}^{*}, \\
J_{d b i}^{*} & =B_{1} P_{I A A H}^{*} d_{i}^{*}-A_{1} P_{I A A H}^{*} b_{i}^{*}, \\
J_{a d i}^{*} & =A_{1} P_{I A A H}^{*} a_{i}^{*}-B_{1} P_{I A A H}^{*} d_{i}^{*}, \\
J_{e h i}^{*} & =B_{1} P_{I A A H}^{*} e_{i}^{*}-A_{1} P_{I A A H}^{*} h_{i}^{*}, \\
J_{h e i}^{*} & =\left(A_{1} P_{I A A H}^{*}+A_{3} P_{P I N 1}^{*}\right) h_{i-1}^{*}-\left(B_{1} P_{I A A H}^{*}+B_{3} P_{P I N 1}^{*}\right) e_{i}^{*}, \\
J_{b e i}^{*} & =A_{1} P_{I A A H}^{*} b_{i}^{*}-B_{1} P_{I A A H}^{*} e_{i}^{*} ;
\end{aligned}
$$

the meaning of the first two subscripts is that, say, $J_{c f i}^{*}$ is the flux from the region which has concentration $c_{i}^{*}$ into the region which has concentration $f_{i}^{*}$.

Both protonated and anionic auxin passively diffuse through the apoplast with diffusivity $D_{a}^{*}$, and therefore the apoplastic diffusive fluxes per unit length are given by

$$
\begin{aligned}
& J_{f k i}^{*}=\frac{2 D_{a}^{*}}{\left(w^{*}+\lambda^{*}\right)}\left(f_{i}^{*}-k_{i}^{*}\right), \quad J_{k g i}^{*}=\frac{2 D_{a}^{*}}{\left(w^{*}+\lambda^{*}\right)}\left(k_{i}^{*}-g_{i}^{*}\right), \\
& J_{g m i}^{*}=\frac{2 D_{a}^{*}}{\left(w^{*}+\lambda^{*}\right)}\left(g_{i}^{*}-m_{i}^{*}\right), \quad J_{m h i}^{*}=\frac{2 D_{a}^{*}}{\left(w^{*}+\lambda^{*}\right)}\left(m_{i}^{*}-h_{i}^{*}\right), \\
& J_{a k i}^{*}=\frac{2 D_{a}^{*}}{\left(l_{i}^{*}+\lambda^{*}\right)}\left(a_{i}^{*}-k_{i}^{*}\right), \quad J_{b m i}^{*}=\frac{2 D_{a}^{*}}{\left(l_{i}^{*}+\lambda^{*}\right)}\left(b_{i}^{*}-m_{i}^{*}\right), \\
& J_{k a i}^{*}=\frac{2 D_{a}^{*}}{\left(l_{i}^{*}+\lambda^{*}\right)}\left(k_{i-1}^{*}-a_{i}^{*}\right), \quad J_{m b i}^{*}=\frac{2 D_{a}^{*}}{\left(l_{i}^{*}+\lambda^{*}\right)}\left(m_{i-1}^{*}-b_{i}^{*}\right) .
\end{aligned}
$$

We assume that there is no flux of auxin from the epidermis into the surrounding environment or from the endodermis into the underlying tissue. Auxin enters the elongation zone at the rootward end; there, we prescribe the cytoplasmic concentrations to be $C^{*}$. At the shootward end, auxin leaves the elongation zone and we adopt zeroconcentration boundary conditions. Thus, we prescribe

$$
\begin{aligned}
& c_{1}^{*}=d_{1}^{*}=e_{1}^{*}=a_{1}^{*}=b_{1}^{*}=C^{*} \text { and } \\
& c_{N+1}^{*}(t)=d_{N+1}^{*}(t)=e_{N+1}^{*}(t)=a_{N+1}^{*}(t)=b_{N+1}^{*}(t)=0 .
\end{aligned}
$$

We note that the boundary conditions and geometry considered does not allow for auxin to be recycled back into the elongation zone via the vasculature tissue at the root centre; this recycling could be addressed in more complex models that incorporate more root tissue layers. We assume for definiteness that initially (at $t^{*}=0$ ) the auxin concentrations are zero throughout the elongation zone.

\subsection{Parameter estimates}

Biologically relevant parameter estimates are discussed in Appendix B and summarised in Table 1. Due to the $\mathrm{pH}$ difference, the proportion of protonated auxin is much higher in the apoplast than the cytoplasms, $A_{1} \gg B_{1}$, which leads to much higher 
Table 1 Dimensional parameters estimates, discussed in Sect. 2.2

\begin{tabular}{lll}
\hline Parameter & Description & Value \\
\hline$l_{T}^{*}$ & Typical cytoplasm length (short cells) & $20 \mu \mathrm{m}$ \\
$l_{T}^{*}$ & Typical cytoplasm length (long cells) & $200 \mu \mathrm{m}$ \\
$w^{*}$ & Cytoplasm width & $10 \mu \mathrm{m}$ \\
$N$ & Number of cells & 20 \\
$\lambda^{*}$ & Apoplast thickness & $0.5 \mu \mathrm{m}$ \\
$P_{I A A H}^{*}$ & Passive membrane permeability & $5.6 \times 10^{-7} \mathrm{~m} \mathrm{~s}^{-1}$ \\
$P_{A U X 1}^{*}$ & Membrane permeability due to AUX1 influx carriers & $5.6 \times 10^{-7} \mathrm{~m} \mathrm{~s}^{-1}$ \\
$P_{P I N}^{*}$ & Membrane permeability due to PIN1 and PIN2 efflux carriers & $5.6 \times 10^{-7} \mathrm{~m} \mathrm{~s}^{-1}$ \\
$P_{P I N 1}^{*}$ & Membrane permeability due to PIN1 efflux carriers & $2.8 \times 10^{-7} \mathrm{~m} \mathrm{~s}^{-1}$ \\
$D_{a}^{*}$ & Diffusion coefficient in the apoplast & $3.2 \times 10^{-11} \mathrm{~m}^{2} \mathrm{~s}^{-1}$ \\
$D_{c}^{*}$ & Diffusion coefficient in the cytoplasm & $6.7 \times 10^{-10} \mathrm{~m}^{2} \mathrm{~s}^{-1}$ \\
$p H_{C}$ & Cytoplasmic pH & 7.2 \\
$p H_{a}$ & Apoplastic pH & 5.3 \\
$p K$ & Dissociation constant for auxin & 4.8 \\
$V^{*}$ & Cell membrane potential & $-0.120 \mathrm{~V}$ \\
$T^{*}$ & Temperature & $300 \mathrm{~K}$ \\
$A_{1}$ & Apoplastic proportion of protonated auxin, (2.1) & 0.24 \\
$B_{1}$ & Cytoplasmic proportion of protonated auxin, (2.1) & 0.004 \\
$A_{2}$ & See (2.4) & 3.57 \\
$B_{2}$ & See (2.4) & 0.045 \\
$A_{3}$ & See (2.4) & 0.034 \\
$B_{3}$ & See (2.4) & 4.68 \\
\hline & & \\
\hline & &
\end{tabular}

passive diffusion into the cytoplasms that out of them. However, even in the apoplast, only $24 \%$ of the auxin is protonated and therefore the remaining $76 \%$ requires influx carriers to enter the cytoplasm. Where influx carriers are present, they produce a flux into the cell that is over ten times the passive diffusion (consider $A_{2} / A_{1}$ ). As noted earlier, the form of the carrier-mediated fluxes, (2.3), leads to the influx carriers creating an efflux and the efflux carriers creating an influx; however, the parameter values suggest that both these fluxes are small $\left(B_{2} \ll 1, A_{3} \ll 1\right)$, as one would hope.

In Sect. 4, we discuss how sensitive our results are to variations in the typical cytoplasm length, membrane permeabilities and apoplast thickness and show that different biological parameter estimates correspond to distinct asymptotic regimes. For the parameter estimates in Table 1, we present the time scales of the key transport processes in Table 2. These confirm that on the cell scale, cytoplasmic diffusion is much faster than both membrane transport and apoplastic diffusion, so that it is appropriate to treat the cytoplasmic concentrations as spatially homogeneous. In addition, these auxin-transport time scales are smaller than the time scales of growth [typical relative cell elongation rates are less than $0.4 \mathrm{~h}^{-1}$ (Swarup et al. 2007)] and cell movement [approximately 2.5 cells enter the elongation zone every hour (Ubeda-Tomás et al. 2009)], justifying our use of a constant array of cells. 
Table 2 Time scales of key transport processes based on the parameters values in Table 1

\begin{tabular}{lll}
\hline Timescale & Formula & Value (s) \\
\hline Membrane transport on the short-cell length scale & $l_{T}^{*} / P_{P I N}^{*}$ & 36 \\
Membrane transport on the long-cell length scale & $l_{T}^{*} / P_{P I N}^{*}$ & 360 \\
Apoplastic diffusion on the short-cell length scale & $l_{T}^{* 2} / D_{a}^{*}$ & 13 \\
Apoplastic diffusion on the long-cell length scale & $l_{T}^{* 2} / D_{a}^{*}$ & 1,300 \\
Cytoplasmic diffusion on the short-cell length scale & $l_{T}^{* 2} / D_{c}^{*}$ & 0.60 \\
Cytoplasmic diffusion on the long-cell length scale & $l_{T}^{* 2} / D_{c}^{*}$ & 60 \\
\hline
\end{tabular}

\subsection{Governing equations}

The rate of change of the number of auxin molecules in each region equals the sum of the various fluxes per unit membrane length into the region multiplied by the appropriate membrane length. Thus, the auxin concentrations can be described by $10 \mathrm{~N}-5$ coupled linear ordinary differential equations for the $10 \mathrm{~N}-5$ unknowns,

$$
\begin{aligned}
w^{*} l_{i}^{*} \frac{\mathrm{d} c_{i}^{*}}{\mathrm{~d} t^{*}} & =w^{*}\left(J_{f c i}^{*}-J_{c f i}^{*}\right)-l_{i}^{*} J_{c a i}^{*}, \\
w^{*} l_{i}^{*} \frac{\mathrm{d} d_{i}^{*}}{\mathrm{~d} t^{*}} & =w^{*}\left(J_{g d i}^{*}-J_{d g i}^{*}\right)+l_{i}^{*}\left(J_{a d i}^{*}-J_{d b i}^{*}\right), \\
w^{*} l_{i}^{*} \frac{\mathrm{d} e_{i}^{*}}{\mathrm{~d} t^{*}} & =w^{*}\left(J_{h e i}^{*}+J_{e h i}^{*}\right)+l_{i}^{*} J_{b e i}^{*}, \\
\lambda^{*} w^{*} \frac{\mathrm{d} f_{i}^{*}}{\mathrm{~d} t^{*}} & =w^{*}\left(J_{c f i}^{*}-J_{f c(i+1)}^{*}\right)-\lambda^{*} J_{f k i}^{*}, \\
\lambda^{*} w^{*} \frac{\mathrm{d} g_{i}^{*}}{\mathrm{~d} t^{*}} & =w^{*}\left(J_{d g i}^{*}-J_{g d(i+1)}^{*}\right)+\lambda^{*}\left(J_{k g i}^{*}-J_{g m i}^{*}\right), \\
\lambda^{*} w^{*} \frac{\mathrm{d} h_{i}^{*}}{\mathrm{~d} t^{*}} & =w^{*}\left(J_{e h i}^{*}-J_{h e(i+1)}^{*}\right)+\lambda^{*} J_{m h i}^{*}, \\
\lambda^{*} l_{i}^{*} \frac{\mathrm{d} a_{i}^{*}}{\mathrm{~d} t^{*}} & =l_{i}^{*}\left(J_{c a i}^{*}-J_{a d i}^{*}\right)+\lambda^{*}\left(J_{k a i}^{*}-J_{a k i}^{*}\right), \\
\lambda^{*} l_{i}^{*} \frac{\mathrm{d} b_{i}^{*}}{\mathrm{~d} t^{*}} & =l_{i}^{*}\left(J_{d b i}^{*}-J_{b e i}^{*}\right)+\lambda^{*}\left(J_{m b i}^{*}-J_{b m i}^{*}\right), \\
\lambda^{*} \frac{\mathrm{d} k_{i}^{*}}{\mathrm{~d} t^{*}} & =J_{a k i}^{*}-J_{k a(i+1)}^{*}+J_{f k i}^{*}-J_{k g i}^{*}, \\
\lambda^{*} \frac{\mathrm{d} m_{i}^{*}}{\mathrm{~d} t^{*}} & =J_{b m i}^{*}-J_{m b(i+1)}^{*}+J_{g m i}^{*}-J_{m h i}^{*},
\end{aligned}
$$

where we impose ( $2.8 \mathrm{a}-\mathrm{c}, \mathrm{g}, \mathrm{h})$ for $i=2,3 \ldots, N$ and $(2.8 \mathrm{~d}-\mathrm{f}, \mathrm{i}, \mathrm{j})$ for $i=1,2, \ldots, N$, and the flux components are given by $(2.5,2.6)$. These equations are subject to boundary conditions, (2.7), which prescribe the concentrations at the centres of the $i=1$ 
and $i=N+1$ cells. Thus, the total length of the tissue is $L^{*}=\left(l_{1}^{*}+l_{N+1}^{*}\right) / 2+\lambda^{*}+$ $\sum_{i=2}^{N}\left(l_{i}^{*}+\lambda^{*}\right)$, and the representative cell length is $l_{T}^{*}=\left(\left(l_{1}^{*}+l_{N+1}^{*}\right) / 2+\sum_{i=2}^{N} l_{i}^{*}\right) / N$, so that $L^{*}=N\left(l_{T}^{*}+\lambda^{*}\right)$.

\section{Model analysis}

The auxin-transport model derived above is based on the cell-scale model of Swarup et al. (2005), who studied auxin transport in the root elongation zone by considering a multi-layered 3D cylinder of cells (a comparison of the model formulations is provided in Appendix C). Having set up their model, Swarup et al. (2005) simulated the governing ordinary differential equations numerically. In contrast, we use analytical methods to simplify the system, (2.8), and gain insight into the underlying auxin dynamics, notably into the tissue-scale transport properties.

\subsection{Nondimensionalisation}

We nondimensionalise the governing equations, (2.8), as follows

$$
\begin{gathered}
t^{*}=\frac{L^{* 2}}{D_{a}^{*}} t, \quad l_{i}^{*}=l_{T}^{*} l_{i}, \\
\left(a_{i}^{*}, b_{i}^{*}, c_{i}^{*}, d_{i}^{*}, e_{i}^{*}, f_{i}^{*}, g_{i}^{*}, h_{i}^{*}, k_{i}^{*}, m_{i}^{*}\right)=C^{*}\left(a_{i}, b_{i}, c_{i}, d_{i}, e_{i}, f_{i}, g_{i}, h_{i}, k_{i}, m_{i}\right) \\
\left(J_{c f i}^{*}, J_{f c i}^{*}, J_{c a i}^{*}, J_{d g i}^{*}, J_{g d i}^{*}, J_{d b i}^{*}, J_{a d i}^{*}, J_{e h i}^{*}, J_{h e i}^{*}, J_{b e i}^{*}\right) \\
=\frac{D_{a}^{*} C^{*}}{l_{T}^{*}+\lambda^{*}}\left(J_{c f i}, J_{f c i}, J_{c a i}, J_{d g i}, J_{g d i}, J_{d b i}, J_{a d i}, J_{e h i}, J_{h e i}, J_{b e i}\right), \\
\left(J_{f k i}^{*}, J_{k g i}^{*}, J_{g m i}^{*}, J_{m h i}^{*}, J_{a k i}^{*}, J_{b m i}^{*}, J_{k a i}^{*}, J_{m b i}^{*}\right) \\
=\frac{D_{a}^{*} C^{*}}{l_{T}^{*}+\lambda^{*}}\left(J_{f k i}, J_{k g i}, J_{g m i}, J_{m h i}, J_{a k i}, J_{b m i}, J_{k a i}, J_{m b i}\right) .
\end{gathered}
$$

As the apoplastic diffusion coefficient is well characterised (Kramer et al. 2007), we choose the time scale based on the rate at which the apoplastic auxin diffuses the length of the tissue. We nondimensionalise the cytoplasm lengths on the typical one, $l_{T}^{*}$, the auxin concentrations on the upstream concentration, $C^{*}$, and the fluxes on the typical apoplastic diffusive flux, (2.6). In the subsequent analysis, we derive two key non-dimensional outputs, namely an effective directed-transport speed, $v_{e f f}$, and an effective diffusivity, $D_{e f f}$ : these correspond to dimensional values of $v_{e f f}^{*}=D_{a}^{*} v_{e f f} /\left(l_{T}^{*}+\lambda^{*}\right) \mathrm{ms}^{-1}$ and $D_{e f f}^{*}=D_{a}^{*} D_{e f f} \mathrm{~m}^{2} \mathrm{~s}^{-1}$.

We introduce the dimensionless parameters

$$
\epsilon=\frac{l_{T}^{*}+\lambda^{*}}{L^{*}}=\frac{1}{N}, \quad \lambda=\frac{\lambda^{*}}{l_{T}^{*}}, \quad w=\frac{w^{*}}{l_{T}^{*}} ;
$$

here $\epsilon$ is the ratio between the typical cell length and the tissue length, while $\lambda$ is the ratio of the apoplast thickness to the typical cytoplasm length and $w$ is the typical 
cytoplasm aspect ratio. In the subsequent analysis, parameter $\epsilon$ is taken to be small: in the Arabidopsis root, the elongation-zone length is $\approx 2,000 \mu \mathrm{m}$ and the cells lengths are 10-200 $\mu \mathrm{m}$ (Swarup et al. 2007), and we therefore set $\epsilon \approx 1 / 20$. The parameter estimates in Tables 1 and 2 suggest that on the cell length scale the rates of carrier-mediated transport and apoplastic diffusion are comparable; therefore we define dimensionless permeability parameters by

$$
\left(P_{I A A H}, P_{A U X 1}, P_{P I N}, P_{P I N 1}\right)=\frac{l_{T}^{*}+\lambda^{*}}{D_{a}^{*}}\left(P_{I A A H}^{*}, P_{A U X 1}^{*}, P_{P I N}^{*}, P_{P I N 1}^{*}\right)
$$

On nondimensionalising in this fashion, the governing equations, (2.8), become

$$
\begin{aligned}
& \frac{\epsilon^{2} w l_{i}}{(1+\lambda)} \frac{\mathrm{d} c_{i}}{\mathrm{~d} t}=w\left(J_{f c i}-J_{c f i}\right)-l_{i} J_{c a i}, \\
& \frac{\epsilon^{2} w l_{i}}{(1+\lambda)} \frac{\mathrm{d} d_{i}}{\mathrm{~d} t}=w\left(J_{g d i}-J_{d g i}\right)+l_{i}\left(J_{a d i}-J_{d b i}\right), \\
& \frac{\epsilon^{2} w l_{i}}{(1+\lambda)} \frac{\mathrm{d} e_{i}}{\mathrm{~d} t}=w\left(J_{h e i}-J_{e h i}\right)+l_{i} J_{b e i}, \\
& \frac{\epsilon^{2} \lambda w}{(1+\lambda)} \frac{\mathrm{d} f_{i}}{\mathrm{~d} t}=w\left(J_{c f i}-J_{f c(i+1)}\right)-\lambda J_{f k i}, \\
& \frac{\epsilon^{2} \lambda w}{(1+\lambda)} \frac{\mathrm{d} g_{i}}{\mathrm{~d} t}=w\left(J_{d g i}-J_{g d(i+1)}\right)+\lambda\left(J_{k g i}-J_{g m i}\right), \\
& \frac{\epsilon^{2} \lambda w}{(1+\lambda)} \frac{\mathrm{d} h_{i}}{\mathrm{~d} t}=w\left(J_{e h i}-J_{h e(i+1)}\right)+\lambda J_{m h i} w, \\
& \frac{\epsilon^{2} \lambda l_{i}}{(1+\lambda)} \frac{\mathrm{d} a_{i}}{\mathrm{~d} t}=l_{i}\left(J_{c a i}-J_{a d i}\right)+\lambda\left(J_{k a i}-J_{a k i}\right), \\
& \frac{\epsilon^{2} \lambda l_{i}}{(1+\lambda)} \frac{\mathrm{d} b_{i}}{\mathrm{~d} t}=l_{i}\left(J_{d b i}-J_{b e i}\right)+\lambda\left(J_{m b i}-J_{b m i}\right), \\
& \frac{\epsilon^{2} \lambda}{(1+\lambda)} \frac{\mathrm{d} k_{i}}{\mathrm{~d} t}=J_{a k i}-J_{k a(i+1)}+J_{f k i}-J_{k g i}, \\
& \frac{\mathrm{d} m_{i}}{\mathrm{~d} t}=J_{b m i}-J_{m b(i+1)}+J_{g m i}-J_{m h i},
\end{aligned}
$$

where $(3.4 \mathrm{a}-\mathrm{c}, \mathrm{g}, \mathrm{h})$ hold for $i=2,3 \ldots, N$ and $(3.4 \mathrm{~d}-\mathrm{f}, \mathrm{i}, \mathrm{j})$ for $i=1,2, \cdots, N$. The fluxes are given by

$$
\begin{gathered}
J_{c f i}=\left(B_{1} P_{I A A H}+B_{2} P_{A U X 1}+B_{3} P_{P I N}\right) c_{i}-\left(A_{1} P_{I A A H}+A_{2} P_{A U X 1}+A_{3} P_{P I N}\right) f_{i}, \\
J_{f c i}=\left(A_{1} P_{I A A H}+A_{2} P_{A U X 1}\right) f_{i-1}-\left(B_{1} P_{I A A H}+B_{2} P_{A U X 1}\right) c_{i}, \\
J_{d g i}=B_{1} P_{I A A H} d_{i}-A_{1} P_{I A A H} g_{i}, \\
J_{g d i}=\left(A_{1} P_{I A A H}+A_{3} P_{P I N}\right) g_{i-1}-\left(B_{1} P_{I A A H}+B_{3} P_{P I N}\right) d_{i},
\end{gathered}
$$




$$
\begin{gathered}
J_{e h i}=B_{1} P_{I A A H} e_{i}-A_{1} P_{I A A H} h_{i}, \\
J_{h e i}=\left(A_{1} P_{I A A H}+A_{3} P_{P I N 1}\right) h_{i-1}-\left(B_{1} P_{I A A H}+B_{3} P_{P I N 1}\right) e_{i}, \\
J_{a d i}=A_{1} P_{I A A H} a_{i}-B_{1} P_{I A A H} d_{i}, \\
J_{c a i}=\left(B_{1} P_{I A A H}+B_{2} P_{A U X 1}\right) c_{i}-\left(A_{1} P_{I A A H}+A_{2} P_{A U X 1}\right) a_{i}, \\
J_{d b i}=B_{1} P_{I A A H} d_{i}-A_{1} P_{I A A H} b_{i}, \quad J_{b e i}=A_{1} P_{I A A H} b_{i}-B_{1} P_{I A A H} e_{i}, \\
J_{f k i}=\frac{2(1+\lambda)}{(w+\lambda)}\left(f_{i}-k_{i}\right), \quad J_{k g i}=\frac{2(1+\lambda)}{(w+\lambda)}\left(k_{i}-g_{i}\right), \quad J_{g m i}=\frac{2(1+\lambda)}{(w+\lambda)}\left(g_{i}-m_{i}\right), \\
J_{m h i}=\frac{2(1+\lambda)}{(w+\lambda)}\left(m_{i}-h_{i}\right), \quad J_{a k i}=\frac{2(1+\lambda)}{\left(l_{i}+\lambda\right)}\left(a_{i}-k_{i}\right), \quad J_{b m i}=\frac{2(1+\lambda)}{\left(l_{i}+\lambda\right)}\left(b_{i}-m_{i}\right), \\
J_{k a i}=\frac{2(1+\lambda)}{\left(l_{i}+\lambda\right)}\left(k_{i-1}-a_{i}\right), \quad J_{m b i}=\frac{2(1+\lambda)}{\left(l_{i}+\lambda\right)}\left(m_{i-1}-b_{i}\right),
\end{gathered}
$$

and from (2.7), the governing equations, (3.4), are subject to the following boundary conditions:

$$
\begin{aligned}
& c_{1}=d_{1}=e_{1}=a_{1}=b_{1}=1, \quad \text { and } \\
& c_{N+1}(t)=d_{N+1}(t)=e_{N+1}(t)=a_{N+1}(t)=b_{N+1}(t)=0 .
\end{aligned}
$$

\subsection{Regrouped governing equations}

The following regroupings of the above governing equations reflect aspects of species conservation and will be used in deriving solvability conditions in the subsequent analysis. In these regroupings, the tissue-scale dynamics are described in terms of either (a) the average auxin concentration at a given position through the tissue, namely

$$
\mathscr{C}_{i}=\frac{l_{i} w\left(c_{i}+d_{i}+e_{i}\right)+\lambda w\left(f_{i}+g_{i}+h_{i}\right)+\lambda l_{i}\left(a_{i}+b_{i}\right)+\lambda^{2}\left(k_{i}+m_{i}\right)}{3 l_{i} w+3 \lambda w+2 \lambda l_{i}+2 \lambda^{2}},
$$

(in other words, the volume-weighted average over the cytoplasmic and apoplastic regions labelled $i$ ), (b) distinct average auxin concentrations in each of the layers, epidermal, cortical and endodermal, given by

$$
\begin{aligned}
C_{i} & =\frac{l_{i} c_{i}+\lambda f_{i}}{l_{i}+\lambda}, \\
D_{i} & =\frac{l_{i} d_{i}+\lambda g_{i}}{l_{i}+\lambda}, \\
E_{i} & =\frac{l_{i} e_{i}+\lambda h_{i}}{l_{i}+\lambda},
\end{aligned}
$$

respectively, or (c) the volume-weighted average in the apoplast, given by

$$
A_{i}=\frac{w\left(f_{i}+g_{i}+h_{i}\right)+l_{i}\left(a_{i}+b_{i}\right)+\lambda\left(k_{i}+m_{i}\right)}{3 w+2 l_{i}+2 \lambda} .
$$


In each case, the dynamics can be described by equations that arise from summing appropriate combinations of the governing equations, (3.4). Thus an equation that governs the average auxin concentration, (3.7), can be found by summing all ten governing equations (3.4) to give

$$
\begin{aligned}
& \frac{\epsilon^{2}\left(3 l_{i} w+3 \lambda w+2 \lambda l_{i}+2 \lambda^{2}\right)}{(1+\lambda)} \frac{\mathrm{d} \mathscr{C}_{i}}{\mathrm{~d} t} \\
& =w\left(J_{f c i}-J_{f c(i+1)}+J_{g d i}-J_{g d(i+1)}+J_{h e i}-J_{h e(i+1)}\right) \\
& \quad+\lambda\left(J_{k a i}-J_{k a(i+1)}+J_{m b i}-J_{m b(i+1)}\right) .
\end{aligned}
$$

In this equation, (3.10), the first grouping of terms on the right-hand side corresponds to the fluxes through the cell files, whereas the second expresses the fluxes through the apoplast. Coupled equations governing the average concentration in each layer, (3.8), arise from summing equations $(3.4 \mathrm{a}-\mathrm{c})$ to $(3.4 \mathrm{~d}-\mathrm{f})$, i.e.

$$
\begin{aligned}
& \frac{\epsilon^{2}\left(l_{i} w+\lambda w\right)}{(1+\lambda)} \frac{\mathrm{d} C_{i}}{\mathrm{~d} t}=w\left(J_{f c i}-J_{f c(i+1)}\right)-l_{i} J_{c a i}-\lambda J_{f k i} \\
& \frac{\epsilon^{2}\left(l_{i} w+\lambda w\right)}{(1+\lambda)} \frac{\mathrm{d} D_{i}}{\mathrm{~d} t}=w\left(J_{g d i}-J_{g d(i+1)}\right)+l_{i}\left(J_{a d i}-J_{b d i}\right)+\lambda\left(J_{k g i}-J_{g m i}\right) \\
& \frac{\epsilon^{2}\left(l_{i} w+\lambda w\right)}{(1+\lambda)} \frac{\mathrm{d} E_{i}}{\mathrm{~d} t}=w\left(J_{h e i}-J_{h e(i+1)}\right)+l_{i} J_{b e i}+\lambda J_{m h i}
\end{aligned}
$$

Here, the first grouping of terms on the right-hand side of each equation describe the flux through the layer and the remaining terms describe the fluxes between the layers. Finally, the average concentration in the apoplast, (3.9), is governed by the summation of $(3.4 d-j)$,

$$
\begin{aligned}
& \frac{\epsilon^{2}\left(3 \lambda w+2 \lambda l_{i}+2 \lambda^{2}\right)}{(1+\lambda)} \frac{\mathrm{d} A_{i}}{\mathrm{~d} t}=w\left(J_{c f i}-J_{f c(i+1)}+J_{d g i}-J_{g d(i+1)}+J_{e h i}-J_{h e(i+1)}\right) \\
& \quad+l_{i}\left(J_{c a i}-J_{a d i}+J_{d b i}-J_{b e i}\right)+\lambda\left(J_{k a i}-J_{k a(i+1)}+J_{m b i}-J_{m b(i+1)}\right),
\end{aligned}
$$

where the first two sets of terms on the right-hand side describe fluxes between the apoplast and the cytoplasms and the final terms describe the fluxes between the apoplast compartments. The above equations will be explained further and referred to frequently throughout Sects. 3.3-3.6.

\subsection{Continuum approximation of the discrete cell-based model}

To determine the dominant tissue-scale dynamics, we take a continuum approximation of the discrete cell-based model, (3.4), which is valid provided the concentration differences between adjacent cells in each layer are small (i.e. transport is fast on the cell scale). In this analysis, the parameter $\epsilon$ is small, as $N \gg 1$, and we consider the concentration differences between the $i$ th and $i+1$ th compartments to be 
$O(\epsilon)$. We let $x^{*}$ measure the dimensional length along the tissue in the shootward direction, which corresponds to a nondimensionalised distance of $x=x^{*} / L^{*}$. Then $c_{i}(t)=c(x, t), l_{i}=l(x), J_{f k i}(t)=J_{f k}(x, t)$, etc. In addition, we restrict our attention to the case in which the cell lengths are slowly varying, thus $l_{i+1}-l_{i}=O(\epsilon)$. Using a Taylor series approximation,

$$
\begin{aligned}
c_{i+1}(t)= & c\left(x+\frac{\epsilon\left(l_{i}+l_{i+1}+2 \lambda\right)}{2(1+\lambda)}, t\right) \\
= & c(x, t)+\epsilon\left(\frac{l+\lambda}{1+\lambda}\right) \frac{\partial c}{\partial x}+\frac{\epsilon^{2}}{2}\left(\frac{l+\lambda}{1+\lambda}\right)^{2} \frac{\partial^{2} c}{\partial x^{2}} \\
& +\frac{\epsilon^{2}(l+\lambda)}{2(1+\lambda)^{2}} \frac{\mathrm{d} l}{\mathrm{~d} x} \frac{\partial c}{\partial x}+O\left(\epsilon^{3}\right),
\end{aligned}
$$

we construct an asymptotic solution for small $\epsilon$, expanding the concentrations as standard perturbation series, for example, $c(x, t)=c_{0}(x, t)+\epsilon c_{1}(x, t)+O\left(\epsilon^{2}\right)$ (henceforth using subscripts to denote order rather than cell number). In the subsequent sections (Sects. 3.4-3.6), we provide a comprehensive analysis of the dynamics with different parameter scalings. We note that although parameters $A_{3}, B_{1}$, and $B_{2}$ are small (Table 1), it instructive for us to consider the distinguished limits with these parameters being $O(1)$ and being $O(\epsilon)$ (see Sect. 3.5.2).

At $O\left(\epsilon^{0}\right)$, the governing equations, (3.4), reduce to a system of algebraic equations that relate the concentrations in each region, namely

$$
\begin{aligned}
0= & w\left(\left(2 A_{1} P_{I A A H}+2 A_{2} P_{A U X 1}+A_{3} P_{P I N}\right) f_{0}\right. \\
& \left.-\left(2 B_{1} P_{I A A H}+2 B_{2} P_{A U X 1}+B_{3} P_{P I N}\right) c_{0}\right) \\
& +l\left(\left(A_{1} P_{I A A H}+A_{2} P_{A U X 1}\right) a_{0}-\left(B_{1} P_{I A A H}+B_{2} P_{A U X 1}\right) c_{0}\right) \\
0= & w\left(\left(2 A_{1} P_{I A A H}+A_{3} P_{P I N}\right) g_{0}-\left(2 B_{1} P_{I A A H}+B_{3} P_{P I N}\right) d_{0}\right) \\
& +l\left(A_{1} P_{I A A H}\left(a_{0}+b_{0}\right)-2 B_{1} P_{I A A H} d_{0}\right) \\
0= & w\left(\left(2 A_{1} P_{I A A H}+A_{3} P_{P I N 1}\right) h_{0}-\left(2 B_{1} P_{I A A H}+B_{3} P_{P I N 1}\right) e_{0}\right) \\
& +l\left(A_{1} P_{I A A H} b_{0}-B_{1} P_{I A A H} e_{0}\right) \\
0= & w\left(\left(2 B_{1} P_{I A A H}+2 B_{2} P_{A U X 1}+B_{3} P_{P I N}\right) c_{0}\right. \\
& \left.-\left(2 A_{1} P_{I A A H}+2 A_{2} P_{A U X 1}+A_{3} P_{P I N}\right) f_{0}\right)+\frac{2 \lambda(1+\lambda)}{(w+\lambda)}\left(k_{0}-f_{0}\right) \\
0= & w\left(\left(2 B_{1} P_{I A A H}+B_{3} P_{P I N}\right) d_{0}-\left(2 A_{1} P_{I A A H}+A_{3} P_{P I N}\right) g_{0}\right) \\
& +\frac{2 \lambda(1+\lambda)}{(w+\lambda)}\left(m_{0}-2 g_{0}+k_{0}\right) \\
0= & w\left(\left(2 B_{1} P_{I A A H}+B_{3} P_{P I N 1}\right) e_{0}-\left(2 A_{1} P_{I A A H}+A_{3} P_{P I N 1}\right) h_{0}\right) \\
& +\frac{2 \lambda(1+\lambda)}{(w+\lambda)}\left(m_{0}-h_{0}\right) \\
0= & l\left(\left(B_{1} P_{I A A H}+B_{2} P_{A U X 1}\right) c_{0}+B_{1} P_{I A A H} d_{0}-\left(2 A_{1} P_{I A A H}+A_{2} P_{A U X 1}\right) a_{0}\right) \\
& +\frac{4 \lambda(1+\lambda)}{(l+\lambda)}\left(k_{0}-a_{0}\right)
\end{aligned}
$$




$$
\begin{aligned}
& 0=l\left(B_{1} P_{I A A H}\left(d_{0}+e_{0}\right)-2 A_{1} P_{I A A H} b_{0}\right)+\frac{4 \lambda(1+\lambda)}{(l+\lambda)}\left(m_{0}-b_{0}\right), \\
& 0=\frac{2}{(l+\lambda)}\left(a_{0}-k_{0}\right)+\frac{1}{(w+\lambda)}\left(f_{0}-2 k_{0}+g_{0}\right), \\
& 0=\frac{2}{(l+\lambda)}\left(b_{0}-m_{0}\right)+\frac{1}{(w+\lambda)}\left(g_{0}-2 m_{0}+h_{0}\right) .
\end{aligned}
$$

[assuming that all the parameters in these expressions are $O(1)$ as $\epsilon \rightarrow 0^{+}$]. These leading-order equations, (3.14), do not form a closed system because they sum to zero due to conservation of mass; we must therefore either consider higher-order terms to find the leading-order solvability condition or appeal to the exact equation $[(3.10)$, (3.11) or (3.12)].

As discussed in Sect. 3.2, the dominant dynamics take one of three different forms. Provided the fluxes between the three layers are sufficiently large (i.e. provided the dominant terms in $(3.14 \mathrm{a}-\mathrm{c})$ are not identical to those in $(3.14 \mathrm{~d}-\mathrm{f}))$, nine of the ten leading-order equations, (3.14), are independent. At leading order, each auxin concentration is proportional to the average auxin concentration, (3.7), and we denote the proportionality constants with a bar, for example, $c_{0}=\bar{c} \mathscr{C}_{0}$. We close the system via summation (3.10) which, on taking the continuum limit, implies that the tissue-scale dynamics can be described by a linear, convection-diffusion equation of the form

$$
\frac{\partial \mathscr{C}_{0}}{\partial t}+v_{e f f} \frac{\partial \mathscr{C}_{0}}{\partial x}=D_{e f f} \frac{\partial^{2} \mathscr{C}_{0}}{\partial x^{2}},
$$

where $v_{\text {eff }}$ and $D_{\text {eff }}$ are the effective velocity and diffusivity respectively and depend on parameters $P_{I A A H}, P_{A U X 1}, P_{P I N}, P_{P I N 1}, w, l$ and $\lambda$ in a fashion that we shall determine below. In this case, auxin distributes itself across the three layers faster than it propagates through the tissue, and so the proportions of auxin in each of the ten regions are in equilibrium on the time scale of interest.

If the fluxes between the three layers are small, Eqs. $(3.14 \mathrm{a}-\mathrm{c})$ are identical to (3.14d-f); only seven of the leading-order equations are then independent and these determine the relationships between the apoplast concentrations and the three cytoplasm concentrations. To close the system, we in effect proceed to next order by considering the summations (3.11). Auxin distributes between the three layers on the same time scale as it propagates the length of the tissue.

In the remaining cases, the fluxes between the apoplast and the cytoplasms are sufficiently small that the dominant flux through the tissue is through the apoplast. The leading-order equations $(3.14 \mathrm{~d}-\mathrm{j})$ give the relationships between the apoplast concentration in each region and the average apoplast concentration, (3.9). Equations $(3.14 \mathrm{a}-\mathrm{c})$ do not apply; instead, the leading-order terms in $(3.4 \mathrm{a}-\mathrm{c})$ provide three ordinary differential equations for the cytoplasm concentrations, which are coupled to an equation for the average apoplast concentration, (3.12).

In the following analysis, we first consider two simple but instructive limit cases: in Sect. 3.4, we take the cell membranes to be impermeable, so that auxin propagates through the tissue by random motion within the apoplast, and in Sect. 3.5, we take the apoplast thickness to be zero and find that the carrier-mediated transport creates 
a directed flux. In other asymptotic regimes, the relative importance of the random and directed fluxes depends on the apoplast thickness, PIN permeabilities and typical cytoplasm aspect ratio; we discuss several distinguished limits in Sect. 3.6.

\subsection{Apoplastic auxin transport}

We first take the cell membranes to be impermeable, $P_{I A A H}=P_{A U X 1}=P_{P I N}=$ $P_{P I N 1}=0$, so that auxin propagates solely through the apoplast and the dynamics are governed by (3.4) with $J_{c f i}=J_{f c i}=J_{c a i}=J_{d g i}=J_{g d i}=J_{d b i}=J_{a d i}=J_{e h i}=$ $J_{h e i}=J_{b e i}=0$ for $i=1,2, \ldots, N$. We initially consider both $w$ and $\lambda$ to be $O(1)$.

In this case, the dynamics can be described in terms of the average apoplast concentration, (3.9), by Eq. (3.12) at $O\left(\epsilon^{2}\right)$. At leading order, the governing equations, $(3.14 \mathrm{~d}-\mathrm{j})$, show that the apoplastic auxin concentrations are equal at each $x$ and $t$ :

$$
\begin{aligned}
A_{0}(x, t) & =a_{0}(x, t)=b_{0}(x, t)=f_{0}(x, t)=g_{0}(x, t)=h_{0}(x, t) \\
& =k_{0}(x, t)=m_{0}(x, t)
\end{aligned}
$$

whereas at first order, the equations, $(3.4 \mathrm{~d}-\mathrm{j})$, provide relationships

$$
f_{1}=g_{1}=h_{1}=k_{1}=m_{1}=\frac{1}{2}\left(\frac{l+\lambda}{1+\lambda}\right) \frac{\partial A_{0}}{\partial x}+a_{1}, \quad a_{1}=b_{1} \text {. }
$$

Although we now have two unknowns, $A_{0}(x, t)$ and $a_{1}(x, t)$, the $a_{1}$ contributions cancel identically in the summation, (3.12), which provides the solvability condition for $A_{0}$

$$
\begin{aligned}
\frac{\partial A_{0}}{\partial t} & =D_{e f f} \frac{\partial^{2} A_{0}}{\partial x^{2}}, \\
D_{e f f} & =\frac{2(l+\lambda)}{(3 w+2 l+2 \lambda)},
\end{aligned}
$$

Thus, with impermeable cell membranes, auxin propagates through the tissue by random motion within the apoplast: this check on the consistency of the formulation is of particular significance since the discretisation of the apoplast (illustrated in Fig. 2) is artificial, in sharp contrast to the cells, which are of course intrinsically discrete objects. The form of the diffusion coefficient, $(3.18 \mathrm{~b})$, reflects the geometry of the tissue and depends on the axial distance travelled through the tissue compared with the length of a typical diffusion pathway; this pathway also involves lateral motion and thus the effective diffusivity reduces if the cell width increases. Considering only a single layer of apoplast (i.e. only regions $a_{i}$ and $k_{i}$ for $i=1,2, \ldots, N$ ), the effective dimensionless diffusion coefficient is unity as expected. Returning to dimensional variables, $(3.1,3.2)$, the effective diffusion coefficient, $(3.18 \mathrm{~b})$, becomes

$$
\frac{2\left(l^{*}+\lambda^{*}\right) D_{a}^{*}}{\left(2 l^{*}+3 w^{*}+2 \lambda^{*}\right)} \approx 1.8 \times 10^{-11} \mathrm{~m}^{2} \mathrm{~s}^{-1}
$$


using a cell length of $l^{*}=20 \mu \mathrm{m}$ and the parameter estimates in Table 1 . Thus, the geometry of the tissue results in an effective tissue-scale diffusivity which is roughly half the diffusivity through the apoplast, $D_{a}^{*}$.

In deriving (3.18), we consider $\lambda$ and $w$ to be $O(1)$. From (3.18), we see that for $\lambda$ small, the dimensionless effective diffusion coefficient is $2 l /(2 l+3 w)$, whereas it tends to one as $w$ tends to zero (as the regions between neighbouring epidermal, cortical and endodermal cytoplasms become negligible).

\subsection{Carrier-mediated auxin transport with a negligible apoplast thickness}

In the second simple case, we consider the apoplast thickness to be zero $(\lambda=0)$, so that auxin propagates solely by crossing cell membranes. In the analysis that follows, we consider the membrane permeabilities, (3.3), to be $O(1)$, in which case auxin propagates on a fast time scale, $\tilde{t}=t / \epsilon$. The same analysis holds provided $\lambda$ is sufficiently small — the necessary $\lambda$ scaling depends on the order of the aspect ratio and membrane permeabilities (see Table 3 for details).

\subsubsection{Carrier-mediated transport through a single cell file with a negligible apoplast thickness}

We first consider the carrier-mediated auxin flux through a single cell file representing, for instance, the epidermis. We suppose that $A_{j}$ and $B_{j}$ are $O(1)$ for $j=1,2,3$, and that auxin does not leave the layer, so that $J_{c a i}=J_{f k i}=0$ for $i=1,2, \ldots, N$. With $\lambda=0$, the average auxin concentration equals the cytoplasmic concentration, i.e. $C_{0}=c_{0},(3.8 \mathrm{a})$, and the leading-order equations, $(3.14 \mathrm{a}, \mathrm{d})$, show the apoplastic concentration to be proportional to this average concentration, such that

$$
f_{0}=\tilde{f} C_{0}, \quad \tilde{f}=\frac{2 B_{1} P_{I A A H}+2 B_{2} P_{A U X 1}+B_{3} P_{P I N}}{2 A_{1} P_{I A A H}+2 A_{2} P_{A U X 1}+A_{3} P_{P I N}} .
$$

As indicated earlier, solvability condition (3.11a) closes the system, giving

$$
\begin{gathered}
\frac{\partial C_{0}}{\partial \tilde{t}}+v_{e p i} \frac{\partial C_{0}}{\partial x}=0, \\
v_{e p i}=\left(A_{1} P_{I A A H}+A_{2} P_{A U X 1}\right) \tilde{f}-\left(B_{1} P_{I A A H}+B_{2} P_{A U X 1}\right) .
\end{gathered}
$$

Therefore, in the epidermis, the efflux carriers create a directed flux in the shootward direction and the analysis provides a formula for the effective auxin velocity, (3.21b). The behaviour at the wave front can be determined by considering a change of variables, $X=\epsilon^{-1 / 2}\left(x-v_{\text {epi }} \tilde{t}\right)$ and proceeding to next order, which gives

$$
\begin{aligned}
\frac{\partial C_{0}}{\partial \tilde{t}} & =D_{e f f}\left(\frac{\partial^{2} C_{0}}{\partial X^{2}}+\frac{1}{l} \frac{\partial l}{\partial X} \frac{\partial C_{0}}{\partial X}\right) \\
D_{e f f} & =\frac{l\left(B_{1} P_{I A A H}+B_{2} P_{A U X 1}+\left(A_{1} P_{I A A H}+A_{2} P_{A U X 1}\right) \tilde{f}\right)}{2} .
\end{aligned}
$$


Thus, the auxin flux has both diffusive and advective components in the frame of reference moving with the wave front (with the advective term only entering if the cell lengths are non-constant). The presence of the diffusion is apparent in the advection-dominated dynamics simulated in Sect. 4; however, to maintain conciseness our analysis focusses only on the leading-order advective terms in the subsequent cases. We note that the diffusive effects enter over the intermediate range of $O\left(\epsilon^{-1 / 2}\right)$ cells, rather than the tissue scale [ $O\left(\epsilon^{-1}\right)$ cells].

Returning to dimensional quantities, the leading-order equations, $(3.20,3.21)$, give

$$
\begin{gathered}
f_{0}^{*}=\tilde{f} C_{0}^{*}, \quad \tilde{f}=\frac{2 B_{1} P_{I A A H}^{*}+2 B_{2} P_{A U X 1}^{*}+B_{3} P_{P I N}^{*}}{2 A_{1} P_{I A A H}^{*}+2 A_{2} P_{A U X 1}^{*}+A_{3} P_{P I N}^{*}}, \\
v_{e p i}^{*}=\left(A_{1} P_{I A A H}^{*}+A_{2} P_{A U X 1}^{*}\right) \tilde{f}-\left(B_{1} P_{I A A H}^{*}+B_{2} P_{A U X 1}^{*}\right) .
\end{gathered}
$$

Following a similar procedure, the apoplastic concentrations in the cortex and endodermis are given respectively by

$$
\begin{aligned}
g_{0} & =\tilde{g} D_{0}, \\
\tilde{g} & =\frac{2 B_{1} P_{I A A H}+B_{3} P_{P I N}}{2 A_{1} P_{I A A H}+A_{3} P_{P I N}}, \\
h_{0} & =\tilde{h} E_{0}, \\
\tilde{h} & =\frac{2 B_{1} P_{I A A H}+B_{3} P_{P I N 1}}{2 A_{1} P_{I A A H}+A_{3} P_{P I N 1}},
\end{aligned}
$$

and the effective auxin velocity through each layer is

$$
\begin{aligned}
& v_{c o r}=-\left(\left(B_{1} P_{I A A H}+B_{3} P_{P I N}\right)-\left(A_{1} P_{I A A H}+A_{3} P_{P I N}\right) \tilde{g}\right), \\
& v_{\text {end }}=-\left(\left(B_{1} P_{I A A H}+B_{3} P_{P I N 1}\right)-\left(A_{1} P_{I A A H}+A_{3} P_{P I N 1}\right) \tilde{h}\right) .
\end{aligned}
$$

In practice, $B_{1}, B_{2}, A_{3}$ are small (see Table 1 ), and the formulae for the relative apoplast concentrations, $(3.20,3.24)$, and the effective velocities, $(3.21 \mathrm{~b}, 3.25)$, simplify to

$$
\begin{aligned}
\tilde{f} & =\frac{B_{3} P_{P I N}}{2 A_{1} P_{I A A H}+2 A_{2} P_{A U X 1}} \\
\tilde{g} & =\frac{B_{3} P_{P I N}}{2 A_{1} P_{I A A H}}, \\
\tilde{h} & =\frac{B_{3} P_{P I N 1}}{2 A_{1} P_{I A A H}}, \\
v_{\text {epi }} & =\frac{B_{3} P_{P I N}}{2}, \\
v_{\text {cor }} & =-\frac{B_{3} P_{P I N}}{2}, \\
v_{\text {end }} & =-\frac{B_{3} P_{P I N 1}}{2} .
\end{aligned}
$$


The continuum limit thus yields that in the cortical and endodermal layers the effective velocities are negative, $(3.26 \mathrm{e}, \mathrm{f})$, as is to be expected: in these layers, the efflux carriers are located on the rootward faces of the cell membranes and create a rootward flux. The apoplast concentrations, $(3.26 \mathrm{a}-\mathrm{c})$, depend on the ratio between the fluxes into and out of the cytoplasms; hence, the relative apoplast concentration is smaller where the efflux-carrier density is smaller or where influx carriers are present. As influx carriers are located on all sides of the epidermal cell membranes, they do not affect the effective tissue-scale velocity, (3.26d). As a result, the effective velocities in the epidermis and cortex are equal (that in the endodermis being smaller due to the lower efflux-carrier density).

In agreement with Mitchison (1980b), the effective velocities, (3.26d-f), are proportional to $P_{P I N}$ or $P_{P I N 1}$ (i.e. the permeability due to the polar components of the membrane transport). These velocities are independent of cell length due to the following two effects cancelling each other out: (i) transport across the cell is taken to be instantaneous, so increasing the cell length in principle increases the rate at which auxin moves through the tissue, (ii) increasing the cell length increases the cell area and hence the number of molecules required to achieve a given concentration within the cell. Similarly, the velocities do not depend on the cell width since the rate at which molecules enter each cell increases with the number of efflux carriers, which is proportional to the cell width; however, increasing the cell width also increases the cell area, requiring more molecules to change the concentration.

If a plant has defective PIN carriers, it may be appropriate to rescale $P_{P I N}$ and $P_{P I N 1}$ to be $O(\epsilon)$, which results in expressions (3.21b) and (3.25) being identically zero. Considering, for example, the epidermis and letting $P_{P I N}=\epsilon \widetilde{P}_{P I N}$, we find that the apoplast concentration is proportional to the average auxin concentration, $C_{0}$, [according to (3.20) with no $P_{P I N}$ contribution], and solvability condition (3.11a) yields

$$
\begin{gathered}
\frac{\partial C_{0}}{\partial t}+v_{e p i} \frac{\partial C_{0}}{\partial x}=D_{e p i} \frac{\partial^{2} C_{0}}{\partial x^{2}}, \quad v_{e p i}=\frac{\widetilde{P}_{P I N}\left(B_{3}-A_{3} \tilde{f}\right)}{2} \\
D_{e p i}=\frac{l\left(B_{1} P_{I A A H}+B_{2} P_{A U X 1}\right)}{2} .
\end{gathered}
$$

Thus, with reduced PIN efflux, the effective tissue-scale auxin flux is described by a convection-diffusion equation. The effective diffusivity is due both to the passive flux (the $P_{I A A H}$ contribution) and to the active influx (the $P_{A U X 1}$ contribution), whereas the effective velocity is created by the polarly distributed efflux carriers. If $P_{P I N}$ is $O\left(\epsilon^{2}\right)$, or $B_{1}$ and $B_{2}$ are $O(\epsilon)$, the relevant term drops out of (3.27), leading respectively to purely diffusive or purely convective auxin propagation.

\subsubsection{Carrier-mediated auxin transport in the three-layer model with a negligible apoplast thickness}

Having considered auxin propagation through a single cell file (Sect. 3.5.1), we now return to the three-layer model of the elongation zone with $\lambda=0$. We first suppose that the remaining parameters are $O(1)$. In this regime, each concentration is proportional 
to the average concentration, (3.7), according to (3.14), and the dynamics are governed by (3.15) with $t$ replaced by $\tilde{t}=t / \epsilon$, and

$$
v_{e f f}=\frac{v_{e p i} \bar{c}+v_{c o r} \bar{d}+v_{e n d} \bar{e}}{3}, \quad D_{e f f}=0,
$$

where $v_{\text {epi }}, v_{\text {cor }}$ and $v_{\text {end }}$ are the effective auxin velocities from the single-layer analysis, (3.21b, 3.25), and their contributions to the effective velocity in the three-layer model depends on the proportion of auxin in each layer. The ratios between the cytoplasmic concentrations, $c_{0}, d_{0}, e_{0}$, and the adjacent apoplastic concentrations, $f_{0}, g_{0}, h_{0}$, are identical to those in the single-layer analysis, $(3.20,3.24 \mathrm{~b}, \mathrm{~d})$ [see $(3.14 \mathrm{~d}-\mathrm{f})]$ and the relative cytoplasmic concentrations in the three layers, $c_{0}, d_{0}, e_{0}$, are determined by $(3.14 \mathrm{a}-\mathrm{c}, \mathrm{g}, \mathrm{h})$, which do not depend on the efflux-carrier permeabilities [using (3.14d-f)].

If we now consider $A_{3}, B_{1}$ and $B_{2}$ to be $O(\epsilon)$, with $w=O(1)$, Eqs. $(3.14 \mathrm{~g}$, h) show that $a_{0}=b_{0}=O(\epsilon)$, because the loss of auxin from the apoplast regions $a$ and $b$ to the cytoplasms is otherwise much greater than its gain via passive membrane diffusion. Then, the leading-order equations $(3.14 \mathrm{a}-\mathrm{c})$ and $(3.14 \mathrm{~d}-\mathrm{f})$ are identical, and equations $(3.14 \mathrm{~d}-\mathrm{j})$ merely determine the relationships between the apoplastic and cytoplasmic concentrations. Equations (3.11) then close the system, which take the form

$$
\begin{aligned}
& \frac{\partial C_{0}}{\partial \tilde{t}}+v_{e p i} \frac{\partial C_{0}}{\partial x}=\frac{1}{w}\left(\left(A_{1} P_{I A A H}+A_{2} P_{A U X 1}\right) \tilde{a}_{0}-\left(\widetilde{B}_{1} P_{I A A H}+\widetilde{B}_{2} P_{A U X 1}\right) C_{0}\right), \\
& \frac{\partial D_{0}}{\partial \tilde{t}}+v_{c o r} \frac{\partial D_{0}}{\partial x}=\frac{1}{w}\left(A_{1} P_{I A A H}\left(\tilde{a}_{0}+\tilde{b}_{0}\right)-2 \widetilde{B}_{1} P_{I A A H} D_{0}\right), \\
& \frac{\partial E_{0}}{\partial \tilde{t}}+v_{\text {end }} \frac{\partial E_{0}}{\partial x}=\frac{1}{w}\left(A_{1} P_{I A A H} \tilde{b}_{0}-\widetilde{B}_{1} P_{I A A H} E_{0}\right),
\end{aligned}
$$

where $\tilde{t}=t / \epsilon, \widetilde{B}_{1}=B_{1} / \epsilon, \widetilde{B}_{2}=B_{2} / \epsilon, \tilde{a}_{0}=a_{0} / \epsilon, \tilde{b}_{0}=b_{0} / \epsilon$ and $v_{\text {epi }}, v_{\text {cor }}$ and $v_{\text {end }}$ are the single-layer velocities, $(3.26 \mathrm{~d}-\mathrm{f})$. Auxin distributes between the three layers on the same time scale as it propagates the length of the tissue, and there is no simple expression for an overall auxin velocity.

For thinner cells, $w=O(\epsilon)$, and with $A_{3}, B_{1}, B_{2}$ being either $O(1)$ or $O(\epsilon)$, auxin distributes rapidly between the three layers. The concentrations are each proportional to the average concentration [according to (3.14)] and the tissue-scale auxin flux is described by the solvability condition (3.15) with $v_{\text {eff }}$ and $D_{\text {eff }}$ given by (3.28). Although with $A_{3}, B_{1}$ and $B_{2}$ being $O(\epsilon)$ the apoplast concentrations $a_{0}$ and $b_{0}$ are $O(\epsilon)$ [as with $w=O(1)$ ], the length of cell membrane facing the adjacent layers is much larger than that facing the neighbouring cells in the same layer, so auxin is able to spread rapidly between the layers [consider the dominant terms in $(3.14 \mathrm{a}-\mathrm{c})]$. The distribution of auxin between the three layers is determined by $(3.14 \mathrm{a}-\mathrm{c}, \mathrm{g}, \mathrm{h})$ [simplified via (3.14d-f)] and depends on the small passive and AUX1-mediated effluxes though the cell membranes facing the neighbouring layers. Thus, if $B_{1} \ll B_{2}$, the influx carriers promote efflux from the epidermal cells which, in view of (3.14), results in small epidermal auxin concentrations. This seems to contradict current thinking in 
auxin-transport studies, and we consider $B_{1}$ and $B_{2}$ to be of the same order throughout this analysis.

Reducing the carrier-mediated influx, $P_{A U X 1}$, does not change the form of the equations governing the dominant tissue-scale dynamics, although the formulae for the proportionality constants and effective velocities no longer contain $P_{A U X 1}$. In contrast, with smaller active efflux, auxin propagates on a slower time scale: considering $w, P_{I A A H}, P_{A U X 1}, A_{3}, B_{1}$ and $B_{2}$ to be $O(1)$ and rescaling $P_{P I N}=$ $\epsilon \widetilde{P}_{P I N}, P_{P I N 1}=\epsilon \widetilde{P}_{P I N 1}$, the concentrations are proportional to the average concentration, which is governed by (3.15) with

$$
\begin{aligned}
& v_{e f f}=\frac{1}{3}\left(\frac{\widetilde{P}_{P I N}\left(B_{3} \bar{c}-A_{3} \bar{f}\right)}{2}-\frac{\widetilde{P}_{P I N}\left(B_{3} \bar{d}-A_{3} \bar{g}\right)}{2}-\frac{\widetilde{P}_{P I N 1}\left(B_{3} \bar{e}-A_{3} \bar{h}\right)}{2}\right) \\
& D_{e f f}=\frac{1}{3}\left(\frac{l\left(B_{1} P_{I A A H}+B_{2} P_{A U X 1}\right) \bar{c}}{2}+\frac{l B_{1} P_{I A A H} \bar{d}}{2}+\frac{l B_{1} P_{I A A H} \bar{e}}{2}\right)
\end{aligned}
$$

Thus, as in the single-layer case, (3.27), the auxin flux is governed by a convectiondiffusion equation; the effective velocity is driven by the weak polar efflux carriers which create a flux that is the same order as the effective diffusive flux due to the non-polar membrane-transport components. The first terms in each expression $(3.30 \mathrm{a}, \mathrm{b})$ represent the fluxes through the epidermis, the second terms the fluxes through the cortex and the third terms the fluxes though the endodermis [see (3.27)].

\subsection{Distinguished limits}

In Sects. 3.6.1-3.6.5, we illustrate the dynamics of the full model by describing five distinguished limits. Limits I-IV are presented because (together with their subcases) they can describe the range of representative asymptotic parameter regimes shown in Table 3, whereas limit V is included as it has particular relevance to the Arabidopsis thaliana PIN mutants (see Sect. 4). As noted in the relevant subsections, each of the simplified cases described in Sects. 3.4 and 3.5 can be captured by one of these distinguished limits.

\subsubsection{Distinguished limit I}

We first consider the case in which auxin spreads rapidly between the three layers, and auxin movement between the cytoplasm and the apoplast is sufficiently fast to create gradients in the leading-order apoplastic concentrations that lead to fluxes that contribute to the leading-order effective velocity. This limit is relevant to the case in which $w, \lambda, P_{I A A H}, P_{A U X 1}, P_{P I N}, P_{P I N 1}, A_{j}$ and $B_{j}$ are all $O(1)$, for $j=1,2,3$. Each auxin concentration is a constant proportion of the average concentration, $\mathscr{C}_{0}(x, t)$, [according to (3.14)], and the tissue-scale flux is governed by (3.15) with $t$ replaced by $\tilde{t}=t / \epsilon$ and 


$$
v_{e f f}=\frac{\left(w(l+\lambda)\left(v_{e p i} \bar{c}+v_{c o r} \bar{d}+v_{e n d} \bar{e}\right)+2 \lambda(1+\lambda)(\bar{k}-\bar{a}+\bar{m}-\bar{b})\right)}{\left(3 w l+3 \lambda w+2 \lambda l+2 \lambda^{2}\right)}, \quad D_{e f f}=0
$$

where $v_{\text {epi }}, v_{\text {cor }}$ and $v_{\text {end }}$ are given by $(3.21 \mathrm{~b}, 3.25)$, and bars denote the proportionality constants. Setting $\lambda=0$ in (3.31), we recover expressions (3.28) that describe the dominant behaviour for carrier-mediated transport. The remaining terms (those depending on $\lambda$ ) arise from the flux through the apoplast; in the impermeable-membranes case (Sect. 3.4), this flux does not contribute to the tissue-scale flux, (3.18), because, for each $i$, the apoplastic concentrations are equal due to the dominance of apoplastic diffusion (i.e. $\bar{k}=\bar{a}=\bar{m}=\bar{b}$ ).

\subsubsection{Distinguished limit II}

We now treat the case in which the movement of auxin between the three layers (through both membrane transport and apoplastic diffusion) is on the same time scale as auxin propagation through the tissue. This distinguished limit corresponds to the parameter regime in which $w, P_{I A A H}, P_{A U X 1}, P_{P I N}, P_{P I N 1}, A_{1}, A_{2}$ and $B_{3}$ are $O(1)$, and we rescale $\lambda=\epsilon \tilde{\lambda}, B_{1}=\epsilon \widetilde{B}_{1}, B_{2}=\epsilon \widetilde{B}_{2}$ and $A_{3}=\epsilon \widetilde{A}_{3}$. At leading order, $(3.14 \mathrm{~g}, \mathrm{~h})$ suggest that $a_{0}$ and $b_{0}$ are $O(\epsilon)$ and $(3.14 \mathrm{a}-\mathrm{c})$ are then equivalent to $(3.14 d-f)$. From (3.11), the dynamics can be described by three coupled partial differential equations

$$
\begin{aligned}
\frac{\partial C_{0}}{\partial \tilde{t}}+v_{e p i} \frac{\partial C_{0}}{\partial x}= & \frac{1}{w}\left(\left(A_{1} P_{I A A H}+A_{2} P_{A U X 1}\right) \tilde{a}_{0}\right. \\
& \left.-\left(\widetilde{B}_{1} P_{I A A H}+\widetilde{B}_{2} P_{A U X 1}\right) C_{0}\right)+\frac{2 \tilde{\lambda}}{l w^{2}}\left(k_{0}-f_{0}\right), \\
\frac{\partial D_{0}}{\partial \tilde{t}}+v_{\text {cor }} \frac{\partial D_{0}}{\partial x}= & \frac{1}{w}\left(A_{1} P_{I A A H}\left(\tilde{a}_{0}+\tilde{b}_{0}\right)-2 \widetilde{B}_{1} P_{I A A H} D_{0}\right) \\
& +\frac{2 \tilde{\lambda}}{l w^{2}}\left(m_{0}-2 g_{0}+k_{0}\right), \\
\frac{\partial E_{0}}{\partial \tilde{t}}+v_{\text {end }} \frac{\partial E_{0}}{\partial x}= & \frac{1}{w}\left(A_{1} P_{I A A H} \tilde{b}_{0}-\widetilde{B}_{1} P_{I A A H} E_{0}\right)+\frac{2 \tilde{\lambda}}{l w^{2}}\left(m_{0}-h_{0}\right),
\end{aligned}
$$

that are coupled via the seven leading-order governing equations $(3.14 \mathrm{~d}-\mathrm{j})$ which relate the apoplastic concentrations to the cytoplasmic ones, $C_{0}, D_{0}$ and $E_{0}$. Here, $\tilde{a}_{0}=a_{0} / \epsilon, \tilde{b}_{0}=b_{0} / \epsilon, \tilde{t}=t / \epsilon$ and $v_{\text {epi }}, v_{\text {cor }}$ and $v_{\text {end }}$ are given by (3.26d-f). Letting $\lambda=0,(3.32)$ reduces to the carrier-mediated-transport case: we obtain (3.29) with $w$ of $O(1)$, and (3.28) if we rescale $w$ to be $O(\epsilon)$.

\subsubsection{Distinguished limit III}

We now consider the case in which the membrane transport creates an effective velocity that is on the same time scale as the effective diffusivity through the apoplast. 
These dynamics occur if $w, \lambda, A_{j}$ and $B_{j}$ are $O(1)$ (for $j=1,2,3$ ), and rescaling $P_{I A A H}=\epsilon \widetilde{P}_{I A A H}, P_{A U X 1}=\epsilon \widetilde{P}_{A U X 1}, P_{P I N}=\epsilon \widetilde{P}_{P I N}$ and $P_{P I N 1}=\epsilon \widetilde{P}_{P I N 1}$.

The dynamics are captured by solvability condition (3.15). As in Sect. 3.4, we must consider both the leading-order and first-order components of the apoplastic concentrations; however, here, the first-order components do not cancel identically in the summation (3.10), since the fluxes across the cell membranes result in spatial variations in the first-order components of the apoplast concentrations, creating apoplastic fluxes that contribute to the leading-order effective tissue-scale velocity. The leadingorder equations, (3.14), show that the concentrations are proportional to the average concentration, $\mathscr{C}_{0}(x, t)$, and that the apoplastic concentrations are equal, (3.16). At next order, we obtain the first-order apoplastic concentrations, via $(3.4 \mathrm{~d}-\mathrm{j})$,

$$
\begin{gathered}
b_{1}=\breve{b} \mathscr{C}_{0}+a_{1}, \quad f_{1}=\breve{f} \mathscr{C}_{0}+\frac{\bar{a}}{2} \frac{\partial \mathscr{C}_{0}}{\partial x}+a_{1}, \quad g_{1}=\breve{g} \mathscr{C}_{0}+\frac{\bar{a}}{2} \frac{\partial \mathscr{C}_{0}}{\partial x}+a_{1}, \\
h_{1}=\breve{h} \mathscr{C}_{0}+\frac{\bar{a}}{2} \frac{\partial \mathscr{C}_{0}}{\partial x}+a_{1}, \quad k_{1}=m_{1}=\breve{k} \mathscr{C}_{0}+\frac{\bar{a}}{2} \frac{\partial \mathscr{C}_{0}}{\partial x}+a_{1},
\end{gathered}
$$

where constants $\breve{b}, \breve{f}, \breve{g}, \breve{h}, \breve{k}$ are solutions of the linear system

$$
\begin{aligned}
0= & w\left(\left(2 B_{1} \widetilde{P}_{I A A H}+2 B_{2} \widetilde{P}_{A U X 1}+B_{3} \widetilde{P}_{P I N}\right) \bar{c}\right. \\
& \left.-\left(2 A_{1} \widetilde{P}_{I A A H}+2 A_{2} \widetilde{P}_{A U X 1}+A_{3} \widetilde{P}_{P I N}\right) \bar{a}\right)+\frac{2 \lambda(1+\lambda)}{(w+\lambda)}(\breve{k}-\breve{f}), \\
0= & w\left(\left(2 B_{1} \widetilde{P}_{I A A H}+2 B_{2} \widetilde{P}_{A U X 1}+B_{3} \widetilde{P}_{P I N}\right) \bar{d}\right. \\
& \left.-\left(2 A_{1} \widetilde{P}_{I A A H}+A_{3} \widetilde{P}_{P I N}\right) \bar{a}\right)+\frac{4 \lambda(1+\lambda)}{(w+\lambda)}(\breve{k}-\breve{g}), \\
0= & w\left(\left(2 B_{1} \widetilde{P}_{I A A H}+B_{3} \widetilde{P}_{P I N 1}\right) \bar{e}\right. \\
& \left.-\left(2 A_{1} \widetilde{P}_{I A A H}+A_{3} \widetilde{P}_{P I N 1}\right) \bar{a}\right)+\frac{2 \lambda(1+\lambda)}{(w+\lambda)}(\breve{k}-\breve{h}), \\
0= & \frac{2 \breve{k}}{l+\lambda}+\frac{1}{w+\lambda}(\breve{f}-2 \breve{k}+\breve{g}), \\
0= & \frac{2}{l+\lambda}(\breve{b}-\breve{k})+\frac{1}{w+\lambda}(\breve{g}-2 \breve{k}+\breve{h}) .
\end{aligned}
$$

(where $\bar{c}, \bar{d}, \bar{e}$ and $\bar{a}$ are again the proportionality constants, e.g. $c_{0}=\bar{c} \mathscr{C}_{0}$ ). The solvability condition, (3.15), then provides the tissue-scale auxin flux with

$$
\begin{aligned}
v_{e f f}= & \frac{1}{\left(3 w l+3 \lambda w+2 \lambda l+2 \lambda^{2}\right)}\left[w(l+\lambda)\left(v_{e p i} \bar{c}+v_{c o r} \bar{d}+v_{e n d} \bar{e}\right)\right. \\
& +2 \lambda(1+\lambda)(2 \breve{k}-\breve{b})], \\
D_{e f f}= & \frac{2 \lambda(l+\lambda) \bar{a}}{\left(3 w l+3 \lambda w+2 \lambda l+2 \lambda^{2}\right)},
\end{aligned}
$$

where $v_{\text {epi }}, v_{\text {cor }}$ and $v_{\text {end }}$ are given by $(3.21 \mathrm{~b}, 3.25)$, and $\breve{k}$ and $\breve{b}$ are solutions of (3.34). If $w=O(\epsilon)$, we find $\breve{b}=\breve{f}=\breve{g}=\breve{h}=\breve{k}=0$ and auxin propagates 
by diffusion through the apoplast, (3.18), with the cytoplasmic concentrations being constant proportions of the average concentration according to $(3.14 \mathrm{a}-\mathrm{c})$ (in contrast with the constant cytoplasmic concentrations in Sect. 3.4).

\subsubsection{Distinguished limit IV}

We now treat the case in which auxin crosses the plasma membrane on the same time scale as apoplastic diffusion the length of the tissue, which occurs with $w, \lambda, A_{j}$ and $B_{j}$ being $O(1)$, for $j=1,2,3$, and rescaling $P_{I A A H}=\epsilon^{2} \widehat{P}_{I A A H}, P_{A U X 1}=$ $\epsilon^{2} \widehat{P}_{A U X 1}, P_{P I N}=\epsilon^{2} \widehat{P}_{P I N}$ and $P_{P I N 1}=\epsilon^{2} \widehat{P}_{P I N 1}$. The leading- and next-order components of the apoplast concentrations are related via (3.16) and (3.17), and the dynamics can be described by Eq. (3.12), which gives

$$
\begin{aligned}
\frac{\partial A_{0}}{\partial t}= & \frac{w(1+\lambda)}{\left(3 \lambda w+2 \lambda l+2 \lambda^{2}\right)}\left(\left(2 B_{1} \widehat{P}_{I A A H}+2 B_{2} \widehat{P}_{A U X 1}+B_{3} \widehat{P}_{P I N}\right) c_{0}\right. \\
& +\left(2 B_{1} \widehat{P}_{I A A H}+B_{3} \widehat{P}_{P I N}\right) d_{0}+\left(2 B_{1} \widehat{P}_{I A A H}+B_{3} \widehat{P}_{P I N 1}\right) e_{0} \\
& \left.-\left(6 A_{1} \widehat{P}_{I A A H}+2 A_{2} \widehat{P}_{A U X 1}+A_{3} \widehat{P}_{P I N}+A_{3} \widehat{P}_{P I N 1}\right) A_{0}\right) \\
& +\frac{l(1+\lambda)}{\left(3 \lambda w+2 \lambda l+2 \lambda^{2}\right)}\left(\left(B_{1} \widehat{P}_{I A A H}+B_{2} \widehat{P}_{A U X 1}\right) c_{0}+2 B_{1} \widehat{P}_{I A A H} d_{0}\right. \\
& \left.+2 B_{1} \widehat{P}_{I A A H} e_{0}-\left(4 A_{1} \widehat{P}_{I A A H}+A_{2} \widehat{P}_{A U X 1}\right) A_{0}\right)+D_{e f f} \frac{\partial^{2} A_{0}}{\partial x^{2}},
\end{aligned}
$$

where $D_{\text {eff }}$ is given by (3.18b). This equation, (3.36), is coupled to three ordinary differential equations that govern the cytoplasm concentrations:

$$
\begin{aligned}
\frac{w l}{(1+\lambda)} \frac{\partial c_{0}}{\partial t}= & w\left(\left(2 A_{1} \widehat{P}_{I A A H}+2 A_{2} \widehat{P}_{A U X 1}+A_{3} \widehat{P}_{P I N}\right) A_{0}\right. \\
& -\left(2 B_{1} \widehat{P}_{I A A H}+2 B_{2} \widehat{P}_{A U X 1}+B_{3} \widehat{P}_{P I N} c_{0}\right) \\
& +l\left(\left(A_{1} \widehat{P}_{I A A H}+A_{2} \widehat{P}_{A U X 1}\right) A_{0}-\left(B_{1} \widehat{P}_{I A A H}+B_{2} \widehat{P}_{A U X 1}\right) c_{0}\right),
\end{aligned}
$$

$$
\begin{aligned}
\frac{w l}{(1+\lambda)} \frac{\partial d_{0}}{\partial t}= & w\left(\left(2 A_{1} \widehat{P}_{I A A H}+A_{3} \widehat{P}_{P I N}\right) A_{0}-\left(2 B_{1} \widehat{P}_{I A A H}+B_{3} \widehat{P}_{P I N}\right) d_{0}\right) \\
& +2 l\left(A_{1} \widehat{P}_{I A A H} A_{0}-B_{1} \widehat{P}_{I A A H} d_{0}\right) \\
\frac{w l}{(1+\lambda)} \frac{\partial e_{0}}{\partial t}= & w\left(\left(2 A_{1} \widehat{P}_{I A A H}+A_{3} \widehat{P}_{P I N 1}\right) A_{0}-\left(2 B_{1} \widehat{P}_{I A A H}+B_{3} \widehat{P}_{P I N 1}\right) e_{0}\right) \\
& +l\left(A_{1} \widehat{P}_{I A A H} A_{0}-B_{1} \widehat{P}_{I A A H} e_{0}\right)
\end{aligned}
$$

If we reduce the order of the permeabilities, (3.36) reduces to that derived in Sect. 3.4, (3.18), where we supposed the membranes to be impermeable. 


\subsubsection{Distinguished limit V}

Finally, we consider the distinguished limit in which the active efflux permeabilities, $P_{P I N}$ and $P_{P I N 1}$, are asymptotically smaller than the passive membrane permeability $P_{I A A H}$, so that the effective diffusivity due to transport across the membranes enters the leading-order governing equation. These dynamics occur if $w, \lambda, P_{I A A H}, P_{A U X 1}, A_{j}$ and $B_{j}$ are $O(1)$, for $j=1,2,3$, and the rescalings $P_{P I N}=$ $\epsilon \widetilde{P}_{P I N}$ and $P_{P I N 1}=\epsilon \widetilde{P}_{P I N 1}$ apply. Auxin distributes rapidly between the layers so that the concentrations are proportional to the average concentration, according to (3.14), and the auxin dynamics are described by solvability condition, (3.15), with

$$
\begin{aligned}
v_{e f f}= & \frac{w(l+\lambda)\left(\widetilde{P}_{P I N}\left(B_{3} \bar{c}-A_{3} \bar{f}\right)-\widetilde{P}_{P I N}\left(B_{3} \bar{d}-A_{3} \bar{g}\right)-\widetilde{P}_{P I N 1}\left(B_{3} \bar{e}-A_{3} \bar{h}\right)\right)}{2\left(3 w l+3 \lambda w+2 \lambda l+2 \lambda^{2}\right)}, \\
D_{e f f}= & \frac{(l+\lambda)}{\left(3 w l+3 \lambda w+2 \lambda l+2 \lambda^{2}\right)}\left(\frac { w ( l + \lambda ) } { 2 ( 1 + \lambda ) } \left(\left(B_{1} P_{I A A H}+B_{2} P_{A U X 1}\right) \bar{c}\right.\right. \\
& \left.\left.+B_{1} P_{I A A H}(\bar{d}+\bar{e})\right)+\lambda(\bar{a}+\bar{b})\right) .
\end{aligned}
$$

Setting $\lambda=0$, we retain the terms that describe the flux through the cell membranes, (3.30), with the remaining components of the effective diffusivity (those that depend on $\lambda$ ) being due to random motion within the apoplast, (3.18).

\subsubsection{Summary of asymptotic regimes}

In Table 3, we consider a range of asymptotic regimes; the behaviour in each is described by one of the distinguished limits described above, possibly after appropriate scaling and simplification. Reducing the influx carrier permeability, $P_{A U X 1}$, does not affect the appropriate distinguished limit, the relevant time scale or the scaling of the concentrations. For weaker active efflux, the order of $P_{P I N}$ and $P_{P I N 1}$ may be smaller than that of $P_{I A A H}$ and $P_{A U X 1}$. The dynamics are then described by either limit IV (Sect. 3.6.4) or limit V (Sect. 3.6.5) depending on the relative strengths of the membrane transport and apoplastic diffusion.

\section{Biological predictions}

Using the parameter estimates in Table 1, we now discuss how the asymptotic analysis presented in Sect. 3 provides insight into the tissue-scale dynamics in the biologically relevant parameter regimes. For both wild type and mutant plants, we discuss the dynamics for short cells and long cells in Sect. 4.1 and 4.2 respectively. These results are summarised in Tables 5 and 6 , in which we provide the dimensional versions of the tissue-scale equations. In both cases, $\epsilon \approx 0.05$, and the relevant parameter scalings are shown in Table 4. 
Table 3 Summary of the auxin dynamics in different asymptotic regimes

\begin{tabular}{|c|c|c|c|}
\hline & $\lambda=O(1)$ & $\lambda=O(\epsilon)$ & $\lambda=O\left(\epsilon^{2}\right)$ \\
\hline \multicolumn{4}{|l|}{$w=O(1)$} \\
\hline \multicolumn{4}{|l|}{$B_{1}, B_{2}, A_{3}=O(1)$} \\
\hline Permeabilities $=O\left(\epsilon^{-1}\right)$ & $\begin{array}{l}\text { Limits } I, I I, I I I^{\mathrm{a}} \\
t=O\left(\epsilon^{2}\right)\end{array}$ & $\begin{array}{l}\text { Limits } I, I I, I I I^{\mathrm{a}} \\
t=O\left(\epsilon^{2}\right)\end{array}$ & $\begin{array}{l}\text { Limits I, II, III } \\
t=O\left(\epsilon^{2}\right)\end{array}$ \\
\hline Permeabilities $=O(1)$ & $\begin{array}{l}\text { Limit I } \\
t=O(\epsilon)\end{array}$ & $\begin{array}{l}\text { Limits } I, I I, I I I^{\mathrm{a}} \\
t=O(\epsilon)\end{array}$ & $\begin{array}{l}\text { Limits I, II, III } \\
t=O(\epsilon)\end{array}$ \\
\hline Permeabilities $=O(\epsilon)$ & $\begin{array}{l}\text { Limit III } \\
t=O(1)\end{array}$ & $\begin{array}{l}\text { Limit I } \\
t=O(1)\end{array}$ & $\begin{array}{l}\text { Limits I,II,III } \\
t=O(1)\end{array}$ \\
\hline Permeabilities $=O\left(\epsilon^{2}\right)$ & $\begin{array}{l}\text { Limit IV } \\
t=O(1)\end{array}$ & $\begin{array}{l}\text { Limit III } \\
t=O\left(\epsilon^{-1}\right)\end{array}$ & $\begin{array}{l}\text { Limit I } \\
t=O\left(\epsilon^{-1}\right)\end{array}$ \\
\hline \multicolumn{4}{|l|}{$B_{1}, B_{2}, A_{3}=O(\epsilon)$} \\
\hline Permeabilities $=O\left(\epsilon^{-1}\right)$ & $\begin{array}{l}\text { Limit II } \\
t=O\left(\epsilon^{2}\right) \\
a, b=O(\epsilon)\end{array}$ & $\begin{array}{l}\text { Limit II }{ }^{\mathrm{b}} \\
t=O\left(\epsilon^{2}\right) \\
a, b=O(\epsilon)\end{array}$ & $\begin{array}{l}\text { Limit II } \\
t=O\left(\epsilon^{2}\right) \\
a, b=O(\epsilon)\end{array}$ \\
\hline Permeabilities $=O(1)$ & $\begin{array}{l}\text { Limit I } \\
t=O(\epsilon)\end{array}$ & $\begin{array}{l}\text { Limit II } \\
t=O(\epsilon) \\
a, b=O(\epsilon)\end{array}$ & $\begin{array}{l}\text { Limit II }{ }^{\mathrm{b}} \\
t=O(\epsilon) \\
a, b=O(\epsilon)\end{array}$ \\
\hline Permeabilities $=O(\epsilon)$ & $\begin{array}{l}\text { Limit III } \\
t=O(1)\end{array}$ & $\begin{array}{l}\text { Limit I } \\
t=O(1)\end{array}$ & $\begin{array}{l}\text { Limit II } \\
t=O(1) \\
a, b=O(\epsilon)\end{array}$ \\
\hline Permeabilities $=O\left(\epsilon^{2}\right)$ & $\begin{array}{l}\text { Limit IV } \\
t=O(1)\end{array}$ & $\begin{array}{l}\text { Limit III } \\
t=O\left(\epsilon^{-1}\right)\end{array}$ & $\begin{array}{l}\text { Limit I } \\
t=O\left(\epsilon^{-1}\right)\end{array}$ \\
\hline \multicolumn{3}{|l|}{$w=O(\epsilon)$} & $B_{1}, B_{2}, A_{3}=O(1)$ \\
\hline Permeabilities $=O\left(\epsilon^{-1}\right)$ & $\begin{array}{l}\text { Limit I } \\
t=O(\epsilon)\end{array}$ & $\begin{array}{l}\text { Limit I } \\
t=O\left(\epsilon^{2}\right)\end{array}$ & $\begin{array}{l}\text { Limits } I, I I, I I I^{\mathrm{a}} \\
t=O\left(\epsilon^{2}\right)\end{array}$ \\
\hline Permeabilities $=O(1)$ & $\begin{array}{l}\text { Limit III } \\
t=O(1)\end{array}$ & $\begin{array}{l}\text { Limit I } \\
t=O(\epsilon)\end{array}$ & $\begin{array}{l}\text { Limit I } \\
t=O(\epsilon)\end{array}$ \\
\hline Permeabilities $=O(\epsilon)$ & $\begin{array}{l}\text { Limits III, IV } \\
t=O(1)\end{array}$ & $\begin{array}{l}\text { Limit III } \\
t=O(1)\end{array}$ & $\begin{array}{l}\text { Limit I } \\
t=O(1)\end{array}$ \\
\hline Permeabilities $=O\left(\epsilon^{2}\right)$ & $\begin{array}{l}\text { Limits III, IV } \\
t=O(1)\end{array}$ & $\begin{array}{l}\text { Limit III } \\
t=O(1)\end{array}$ & $\begin{array}{l}\text { Limit III } \\
t=O\left(\epsilon^{-1}\right)\end{array}$ \\
\hline \multicolumn{4}{|l|}{$B_{1}, B_{2}, A_{3}=O(\epsilon)$} \\
\hline Permeabilities $=O\left(\epsilon^{-1}\right)$ & $\begin{array}{l}\text { Limit I } \\
t=O(\epsilon) \\
\bar{c}, \bar{d}, \bar{e}=O\left(\epsilon^{-1}\right)\end{array}$ & $\begin{array}{l}\text { Limit I } \\
t=O\left(\epsilon^{2}\right) \\
\bar{a}, \bar{b}=O(\epsilon)\end{array}$ & $\begin{array}{l}\text { Limits I, II, III } \\
t=O\left(\epsilon^{2}\right) \\
\bar{a}, \bar{b}=O(\epsilon)\end{array}$ \\
\hline Permeabilities $=O(1)$ & $\begin{array}{l}\text { Limit I } \\
t=O(\epsilon) \\
\bar{c}, \bar{d}, \bar{e}=O\left(\epsilon^{-1}\right)\end{array}$ & $\begin{array}{l}\text { Limit I } \\
t=O(\epsilon) \\
\bar{a}, \bar{b}=O(\epsilon)\end{array}$ & $\begin{array}{l}\text { Limit I } \\
t=O(\epsilon) \\
\bar{a}, \bar{b}=O(\epsilon)\end{array}$ \\
\hline
\end{tabular}


Table 3 continued

\begin{tabular}{llll}
\hline & $\lambda=O(1)$ & $\lambda=O(\epsilon)$ & $\lambda=O\left(\epsilon^{2}\right)$ \\
\hline \multirow{2}{*}{ Permeabilities $=O(\epsilon)$} & Limit III & Limit I & Limit I \\
& $t=O(1)$ & $t=O(1)$ & $t=O(1)$ \\
& $a, b, f, g$, & $\bar{a}, \bar{b}, \bar{f}, \bar{g}$, & $\bar{a}, \bar{b}=O(\epsilon)$ \\
Permeabilities, $O\left(\epsilon^{2}\right)$ & $h, k, m=O(\epsilon)$ & $\bar{h}, \bar{k}, \bar{m}=O(\epsilon)$ & \\
& Limit IV & Limit III & Limit I \\
& $t=O(1)$ & $t=O\left(\epsilon^{-1}\right)$ & $t=O\left(\epsilon^{-1}\right)$ \\
& $a, b, f, g$, & $a, b, f, g$, & $\bar{a}, \bar{b}, \bar{f}, \bar{g}$, \\
& $h, k, m=O(\epsilon)$ & $h, k, m=O(\epsilon)$ & $\bar{h}, \bar{k}, \bar{m}=O(\epsilon)$ \\
\hline
\end{tabular}

The distinguished limits are highlighted in bold and the remaining regimes are subcases of the limits specified. Italic text is used to highlight the regimes in which the dynamics are identical to those with $\lambda=0$; we label with ${ }^{\mathrm{a}}$ and ${ }^{\mathrm{b}}$ the regimes that corresponding to (3.28) and (3.29) respectively

Table 4 Summary of the biologically relevant nondimensionalised parameters

These values arise from the dimensional parameter estimates presented in Table 1

\begin{tabular}{lll}
\hline & $\begin{array}{l}\text { Short cells, } \\
l_{T}^{*}=20 \mu \mathrm{m}\end{array}$ & $\begin{array}{l}\text { Long cells, } \\
l_{T}^{*}=200 \mu \mathrm{m}\end{array}$ \\
\hline$A_{1}$ & 0.24 & 0.24 \\
$A_{2}$ & 3.57 & 3.57 \\
$A_{3}$ & $0.68 \epsilon$ & $0.68 \epsilon$ \\
$B_{1}$ & $\epsilon$ & $\epsilon$ \\
$B_{2}$ & $0.9 \epsilon$ & $0.9 \epsilon$ \\
$B_{3}$ & 4.68 & 4.68 \\
$w$ & 0.5 & $\epsilon$ \\
$\lambda$ & $\epsilon / 2$ & $\epsilon^{2}$ \\
$P_{I A A H}$ & 0.36 & $0.18 / \epsilon$ \\
$P_{A U X 1}$ & 0.36 & $0.18 / \epsilon$ \\
$P_{P I N}$ & 0.36 & $0.18 / \epsilon$ \\
$P_{P I N 1}$ & 0.18 & $0.088 / \epsilon$ \\
\hline
\end{tabular}

\subsection{Short cells}

From Tables 3 and 4, the short-cell dynamics are described by distinguished limit II (Sect. 3.6.2): auxin distributes between the three layers on the same time scale as it propagates through the tissue and the leading-order cytoplasm concentrations are governed by three coupled first-order partial differential equations, (3.32) with $(3.14 \mathrm{~d}-\mathrm{j})$. As the governing equations are first order, we solve numerically in terms of characteristic variables. The $C_{0}$ equation, (3.32a) requires a boundary condition at $x=0$, (3.6a), whereas the $D_{0}$ and $E_{0}$ equations, $(3.32 \mathrm{~b}, \mathrm{c})$, depend on the boundary conditions at $x=1,(3.6 \mathrm{~b})$ (as the velocity in these layers is in the rootward direction). As shown in Fig. 3, solutions of the asymptotic (reduced) model faithfully reproduce numerical solutions of the discrete cell-based model, except at the front of the wave 
(a) $t=0.2 \epsilon$

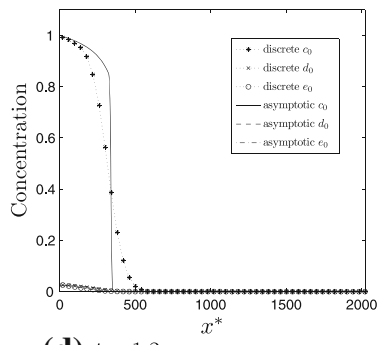

(d) $t=1.2 \epsilon$

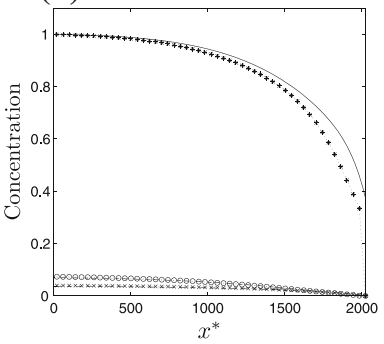

(b) $t=0.4 \epsilon$

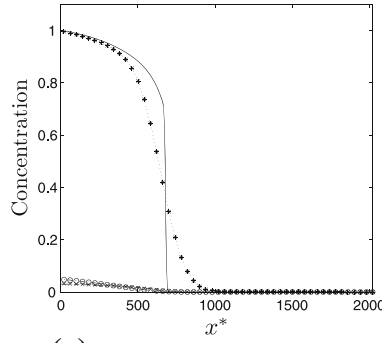

(e) $t=1.6 \epsilon$

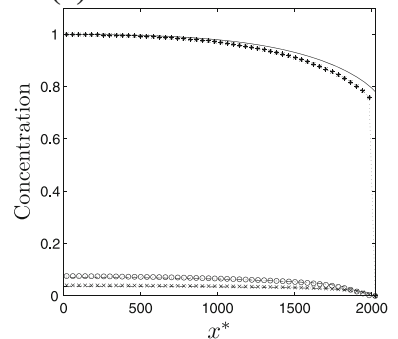

(c) $t=0.8 \epsilon$

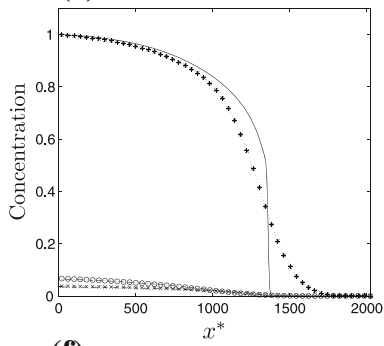

(f) $t=2 \epsilon$

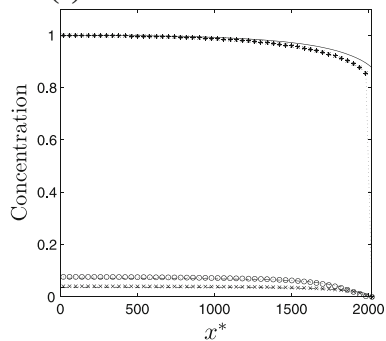

Fig. 3 Short-cell dynamics. Solutions of the reduced model, (3.32) with (3.14d-j), are compared to solutions of the discrete cell-based model (3.4), with boundary conditions (3.6) and the short-cell parameter values in Table 4. a $t=0.2 \epsilon, \mathbf{b} t=0.4 \epsilon, \mathbf{c} t=0.8 \epsilon, \mathbf{d} t=1.2 \epsilon, \mathbf{e} t=1.6 \epsilon$ and $\mathbf{f} t=2 \epsilon$. We use $\epsilon=0.01$ for illustrative purposes

of auxin; accurately predicting the dynamics at the wave front would require consideration of an inner rescaling, as described in the single-cell-file analysis in Sect. 3.5.1. If we were to use no-flux boundary conditions at $x=1$ rather than prescribing a zero concentration there, the early-time dynamics are the same as those for zero-concentration boundary conditions, (3.6b), until the wave front reaches $x=1$ (results not shown).

The reduced model, (3.32) with (3.14d-j), clearly shows how the parameter values affect the tissue-scale dynamics. The tissue-scale auxin flux propagates through the epidermis, and the position of the wave front is therefore determined by the effective velocity in this layer, $B_{3} P_{P I N} / 2$. Auxin gradually spreads into the cortex and endodermis and the distribution between the three layers is affected by the permeabilities due to both the PIN carriers (as these determine the proportion of auxin in the apoplast) and the AUX1 carriers (as these reduce the amount of auxin that leaves the epidermis to enter the cortex and endodermis).

To represent a pin 2 mutant, we would reduce $P_{P I N}$ by half resulting in $v_{e p i}$ and $v_{c o r}$ being halved in the reduced model, whereas for a pinl mutant, both $P_{P I N}$ is halved and $P_{P I N 1}$ is approximately zero so that $v_{e p i}$ and $v_{c o r}$ are halved and $v_{\text {end }} \approx 0$ [see (3.26d-f)]. In both cases, the dynamics are still governed by (3.32) with $(3.14 \mathrm{~d}-\mathrm{j})$ but the wave propagates more slowly due to the reduced epidermal velocity. For a double pinlpin2 mutant, it would be appropriate to set $P_{P I N}$ and $P_{P I N 1}$ to be $O(\epsilon)$ or smaller, resulting in dynamics that are captured by a subcase of distinguished limit $\mathrm{V}$ (Sect. 3.6.5). In this regime, auxin propagates through the tissue more slowly, enabling it to spread between the three layers on a faster time scale than the tissue-scale auxin 
(a) $t=0.2 \epsilon$

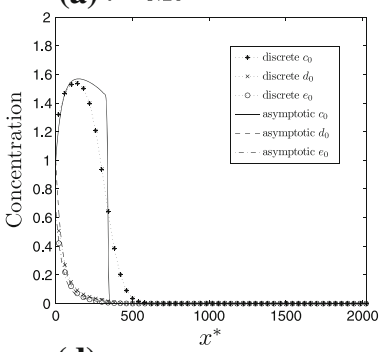

(d) $t=1.2 \epsilon$

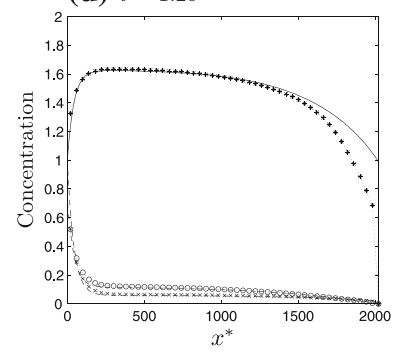

(b) $t=0.4 \epsilon$

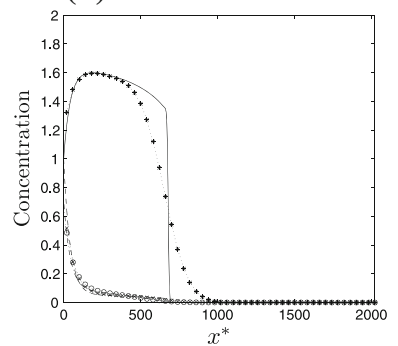

(e) $t=1.6 \epsilon$

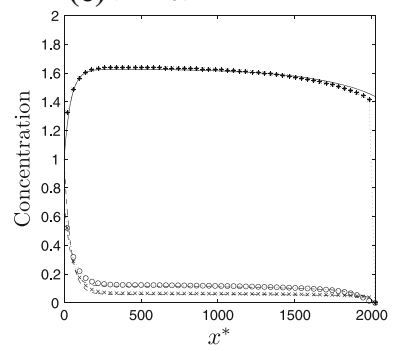

(c) $t=08 \epsilon$

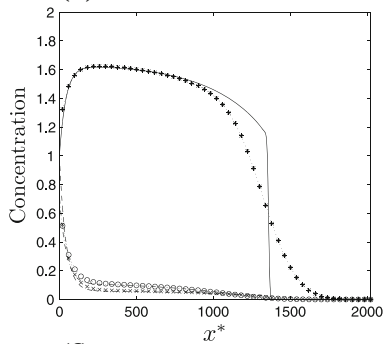

(f) $t=2 \epsilon$

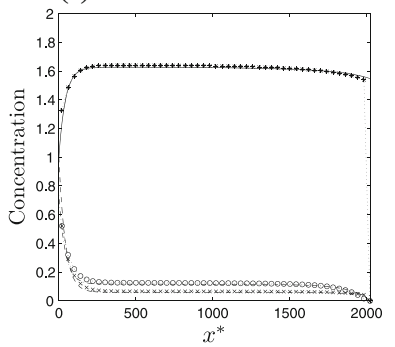

Fig. 4 Short-cell dynamics for a model in which the cortical cells have PIN efflux carriers on the shootward face of their membrane. Solutions of the reduced model, (3.32) with (3.14d-j), are compared to solutions of the discrete cell-based model (3.4), with boundary conditions (3.6) and the short-cell parameter values in Table 4. a $t=0.2 \epsilon, \mathbf{b} t=0.4 \epsilon, \mathbf{c} t=0.8 \epsilon, \mathbf{d} t=1.2 \epsilon, \mathbf{e} t=1.6 \epsilon$ and $\mathbf{f} t=2 \epsilon$. We use $\epsilon=0.01$ for illustrative purposes

flux; this results in the leading order concentrations being proportional to the average auxin concentration, (3.7), according to (3.14). With the parameter estimates in Table 4 and setting $P_{P I N}=0.36 \epsilon$, and $P_{P I N 1}=0.18 \epsilon$, for example, we find that the concentrations in the cortex and endodermis are equal and about a tenth of the epidermal concentrations ( $\bar{c}=2.53$ and $\bar{d}=\bar{e}=0.248$ ). The dominant terms in (3.38) then show that the effective diffusivity is negligible and auxin propagates slowly by almost directed motion.

Following Swarup et al. (2005), we have based our analysis on data from Blilou et al. (2005) in which the elongation-zone cortical cells have efflux carriers on their rootward side; however, other sources suggest that efflux carriers are present instead on the shootward face of these cortical cells (Peer et al. 2004). This is of particular interest as a recent experimental study (Rahman et al. 2010) (focussing on the meristem) suggests that changing the direction of the cortical cells' efflux carriers significantly alters the auxin distribution and gravitropic response. It is straightforward to see how changing the location of these efflux carriers affects the analytical results: in the single-layer analysis, the effective velocity in the cortex becomes $v_{c o r}=B_{3} P_{P I N} / 2$, and we accordingly use this formula for $v_{c o r}$ in place of (3.26e) when evaluating the dynamics of the three-layer model, (3.32) with $(3.14 \mathrm{~d}-\mathrm{j})$. Solutions of both the discrete and the reduced models show that changing the location of the cortical efflux carriers significantly increases the auxin concentrations within all three layers of the tissue (compare Figs. 3, 4). 


\subsection{Long cells}

From Tables 3 and 4, the long-cell dynamics are illustrated by a subcase of distinguished limits I, II and III (Sects. 3.6.1-3.6.3) and are captured by the limit in which fluxes between the apoplast regions are negligible, (3.15) with (3.28). Auxin spreads rapidly between the three layers and maintains this equilibrium-partitioned lateral distribution as it propagates through the tissue, resulting in leading-order concentrations that are proportional to the average auxin concentration, according to (3.14). Using the parameter estimates in Table 4, the proportionality constants are

$$
\begin{gathered}
\bar{c}=2.4, \quad \bar{d}=\bar{e}=0.29, \quad \bar{a}=\bar{b}=0.012, \quad \bar{f}=1.5, \\
\bar{g}=2.8, \quad \bar{h}=1.4, \quad \bar{k}=2.1, \quad \bar{m}=2.1 ;
\end{gathered}
$$

the concentrations in the cortex and endodermis are equal and approximately a tenth of the epidermal concentrations. Auxin propagates through the tissue by a directed flux driven by the efflux carriers on a fast time scale, $t=O\left(\epsilon^{2}\right)$. The effective diffusivity is negligible, and the estimates in Table 4 suggest that the effective velocity is $v_{\text {eff }}=0.27 / \epsilon$, which corresponds to a dimensional value of $8.6 \times 10^{-7} \mathrm{~m} \mathrm{~s}^{-1}$ or $0.31 \mathrm{~cm} \mathrm{~h}^{-1}$.

The leading-order dynamics are described by a single first-order partial differential equation, (3.15) with (3.28) and (3.14), and therefore we prescribe only the upstream boundary conditions (3.6a). A boundary layer exists near $x=0$; in this region, the continuum approximation is not appropriate and we must consider the dominant terms in the discrete equations, $(3.4 \mathrm{a}-\mathrm{c}, \mathrm{g}, \mathrm{h})$. We find that transport between neighbouring cells within each layer balances transport between the three layers, according to

$$
\begin{aligned}
0= & \frac{\tilde{w} B_{3} P_{P I N}}{2}\left(c_{j-1}-c_{j}\right)+l\left(\left(A_{1} P_{I A A H}+A_{2} P_{A U X 1}\right) a_{j}\right. \\
& \left.-\left(\widetilde{B}_{1} P_{I A A H}+\widetilde{B}_{2} P_{A U X 1}\right) c_{j}\right), \\
0= & \frac{\tilde{w} B_{3} P_{P I N}}{2}\left(d_{j+1}-d_{j}\right)+l\left(A_{1} P_{I A A H}\left(a_{j}+b_{j}\right)-\widetilde{B}_{1} P_{I A A H} d_{j}\right), \\
0= & \frac{\tilde{w} B_{3} P_{P I N 1}}{2}\left(e_{j+1}-e_{j}\right)+l\left(A_{1} P_{I A A H} b_{j}-\widetilde{B}_{1} P_{I A A H} e_{j}\right),
\end{aligned}
$$

where

$$
a_{j}=\frac{\left(\widetilde{B}_{1} P_{I A A H}+\widetilde{B}_{2} P_{A U X 1}\right) c_{j}+\widetilde{B}_{1} P_{I A A H} d_{j}}{2 A_{1} P_{I A A H}+A_{2} P_{A U X 1}}, \quad b_{j}=\frac{\widetilde{B}_{1} P_{I A A H}\left(d_{j}+e_{j}\right)}{2 A_{1} P I A A H},
$$

for $j=2,3, \ldots, j_{\text {max }}, B_{1}=\epsilon \widetilde{B}_{1}, B_{2}=\epsilon \widetilde{B}_{2}$ and $w=\epsilon \tilde{w}$, and where $j_{\text {max }}$ labels the cell at the downstream end of the boundary layer. Thus, we have a system of quasisteady equations to solve for the concentrations in the boundary layer; we prescribe $c_{1}=1$ and match to the $x=O(1)$ dynamics by setting $d_{j_{\max }+1}=d_{j_{\max }}, e_{j_{\max }+1}=$ $e_{j_{\max }}$ (ensuring that the concentrations in each layer have no spatial dependence at the 
(a) $t=0.5 \epsilon^{2}$

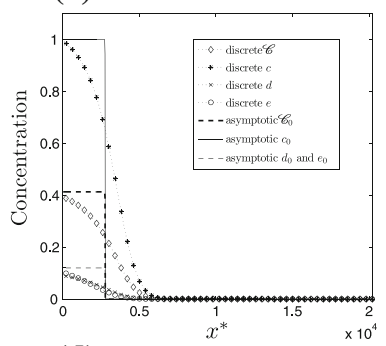

(d) $t=3 \epsilon^{2}$

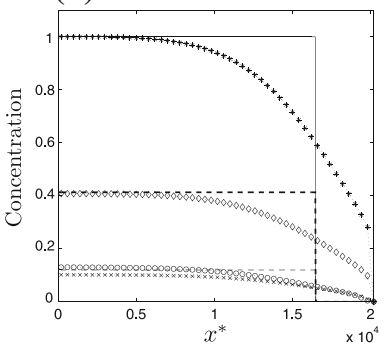

(b) $t=\epsilon^{2}$

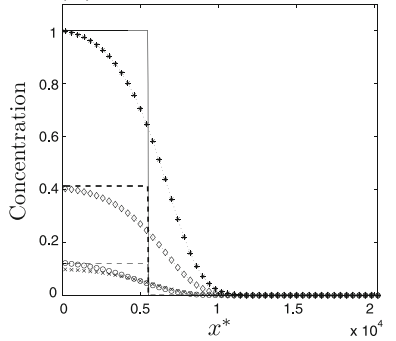

(e) $t=4 \epsilon^{2}$

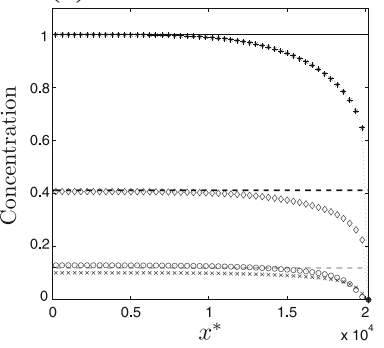

(c) $t=2 \epsilon^{2}$

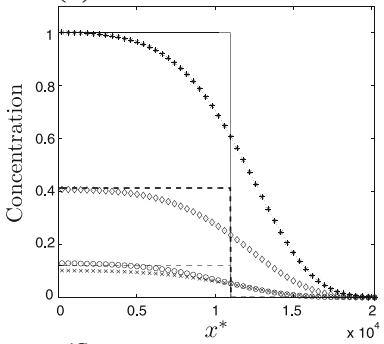

(f) $t=5 \epsilon^{2}$

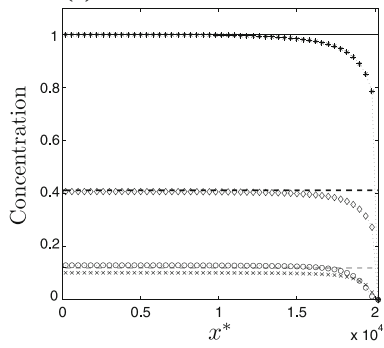

Fig. 5 Long-cell dynamics. Solutions of the reduced model, (3.15) with (3.28) and (3.14), are compared to solutions of the discrete cell-based model (3.4) for the long-cell parameter values in Table 4. We use $\epsilon=0.01$ for illustrative purposes

downstream end of the boundary layer). We see at once that the leading-order dynamics are not affected by the prescribed concentrations $d_{1}$ and $e_{1}$. Solving (4.2) numerically with $j_{\max }=5$ and the parameter estimates in Table 4 , we find that $\mathscr{C}=0.41$ at the downstream end of the boundary layer; doubling the value of $j_{\text {max }}$ does not affect this result. The numerical solutions show that in this case the boundary layer turns out to be particularly narrow so that in practice we have throughout the domain that $c \approx c_{1}$ and $\mathscr{C} \approx c_{1} / \bar{c}$ [in terms of (4.2) we find that for all $j_{\max }, d_{j_{\max }} \approx d_{2}$ and $e_{j_{\max }} \approx e_{2}$ so that the summation of (4.2) gives $\left.c_{j_{\max }}=c_{1}\right]$. We can now capture the dynamics for $x=O(1)$ by setting $\mathscr{C}_{0}(0, t)=0.41$ in the reduced model. Figure 5 demonstrates excellent agreement between solutions of the reduced and discrete models, except in the final few cells at the shootward end of the elongation zone where the discrete model solutions show the concentrations rapidly reducing to zero. We would need to consider an inner solution to capture these $x=1$ boundary-layer dynamics in the reduced model.

In this regime, the formulae clearly show how the permeability parameters affect the dominant dynamics. The relative auxin concentrations are not affected by the effluxcarrier permeabilities, whereas reducing the permeability due to the influx carriers causes the proportion in the epidermis to decrease and the proportions in the cortex and endodermis to increase (Fig. 6a). The proportion in each layer affects the effective velocity as the overall shootward flux depends on the difference between the shootward flux in the epidermis and the rootward flux in the cortex and endodermis, therefore, reducing $P_{A U X 1}$ reduces the overall shootward velocity (Fig. 6b, c). In an auxl mutant, $P_{A U X 1}$ would be an order smaller and would not enter the dominant balance- 
(a)

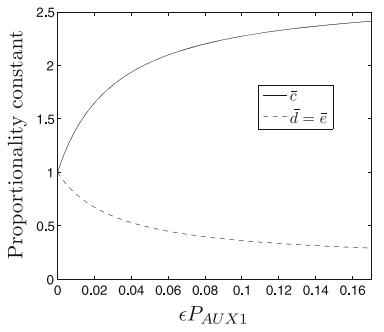

(b)

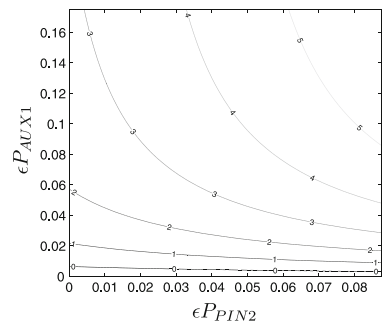

(c)

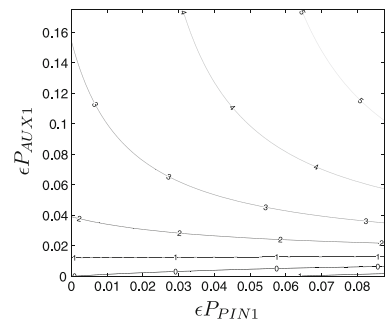

Fig. 6 The influence of the permeability parameters on the auxin distribution and effective velocity for long cells, using $\epsilon=0.05$ and writing $P_{P I N 2}=P_{P I N}-P_{P I N 1}$. In the long-cell regime, $\epsilon P_{P I N}, \epsilon P_{P I N 1}$ and $\epsilon P_{A U X 1}$ are $O(1)$. a The influence of $P_{A U X 1}$ on the proportion of auxin in the three layers, $\mathbf{b}$ the influence of $P_{A U X 1}$ and $P_{P I N 2}$ on the effective velocity for $P_{P I N 1}=0.088 / \epsilon, \mathbf{c}$ the influence of $P_{A U X 1}$ and $P_{P I N 1}$ on the effective velocity, for $P_{P I N 2}=0.088 / \epsilon$

the cytoplasmic concentrations across the three layers are approximately equal and the effective tissue-scale velocity is in the rootward (i.e. reverse) direction (Fig. 6a-c).

As expected, reducing the permeabilities due to either the PIN1 or PIN2 carriers also causes a decrease in the effective velocity provided influx carriers are present, $P_{A U X 1}=O(1 / \epsilon)$ (Fig. 6b, c). Changing the efflux-carrier permeabilities to represent the single pin1 and pin2 mutants (as described in Sect. 4.1), the dynamics are also described by (3.15) with (3.28). In both cases, the reduced model predicts a slow directed shootward flow, provided $P_{A U X 1}$ is $O\left(\epsilon^{-1}\right)$ (Fig. 6b, c). For a pin1pin2 double mutant, with both $P_{P I N}$ and $P_{P I N 1}$ being $O(1)$ or smaller, the dynamics are represented by a subcase of distinguished limit $\mathrm{V}$ in which apoplastic diffusion is negligible (as $\lambda$ is small). Auxin rapidly distributes between the three layers and propagates though the tissue on a slow time scale, potentially with both advective and diffusive flux components (depending on the order of $P_{P I N}$ and $P_{P I N 1}$ ).

As for the short cells, if the cortical cells have efflux carriers on their shootward face, rather than on the rootward one, we see significant changes in the auxin concentrations. The leading-order equations, (3.14), show that the location of the efflux carriers does not affect the proportion of auxin in each layer. However, the dynamics in the boundary layer near $x=0$ are now governed by (4.2) with (4.2b) replaced by

$$
\begin{aligned}
& 0=\frac{\tilde{w} B_{3} P_{P I N}}{2}\left(d_{j-1}-d_{j}\right)+l\left(A_{1} P_{I A A H}\left(a_{j}+b_{j}\right)-\widetilde{B}_{1} P_{I A A H} d_{j}\right) \\
& \quad \text { for } j=2,3, \ldots j_{\text {max }} .
\end{aligned}
$$

We solve this system of linear equations (4.2a, c, 4.4) numerically, with $c_{1}=d_{1}=$ $1, e_{j_{\max }+1}=e_{j_{\max }}, j_{\max }=20$ and the parameter estimates in Table 4 , and find that $\mathscr{C}=0.69$ at the downstream end of the boundary layer. Thus, the average auxin concentration is much higher with the cortical efflux carriers in the shootward direction than in the rootward direction. We find that the spatial extent of the boundary layer is larger in this case, with shootward cortical efflux carriers, than in the case with rootward cortical efflux carriers described by (4.2) (results not shown). Solving the reduced model with $\mathscr{C}_{0}(0, t)=0.69$, we obtain excellent agreement with solutions of 
(a) $t=0.5 \epsilon^{2}$

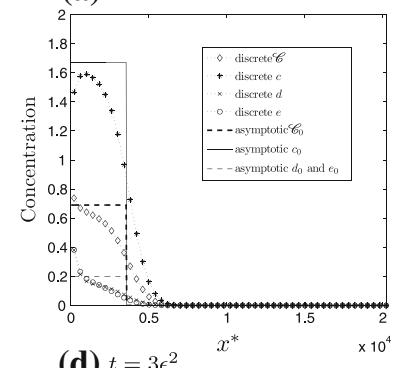

(d) $t=3 \epsilon^{2}$

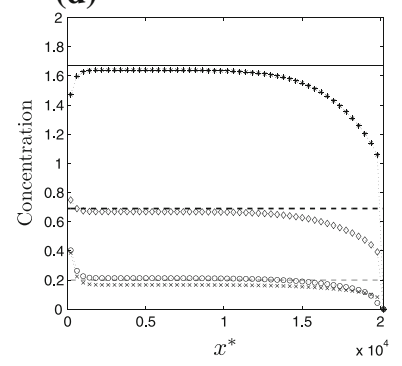

(b) $t=\epsilon^{2}$

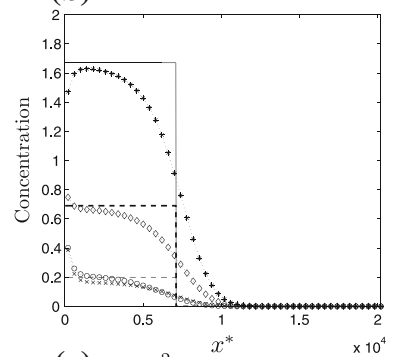

(e) $t=4 \epsilon^{2}$

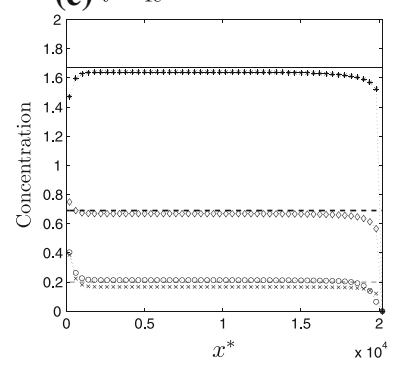

(c) $t=2 \epsilon^{2}$
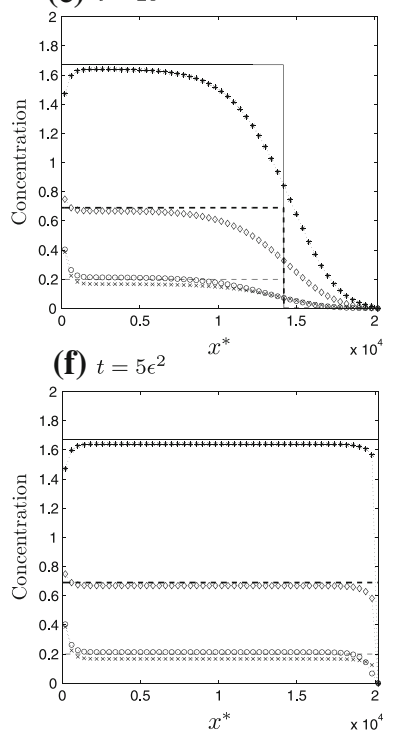

Fig. 7 Long-cell dynamics for a model in which the cortical cells have PIN efflux carriers on the shootward face of their membrane. Solutions of the reduced model, (3.15) with (3.28) and (3.14), are compared to solutions of the discrete cell-based model (3.4) for the long-cell parameter values in Table 4. We use $\epsilon=0.01$ for illustrative purposes

the discrete system (Fig. 7). Comparing Figs. 5 and 7, we see that the location of the cortical efflux carriers significantly affects the amount of auxin that enters the tissue, with shootward efflux carriers creating higher auxin concentrations in each layer.

\subsection{Model variations}

As noted in Sect. 2.2, we use an apoplast thickness of $\lambda^{*}=0.5 \mu \mathrm{m}$ from Swarup et al. (2005); however, recent measurements suggest a lower estimate of $\lambda^{*}=0.14 \mu \mathrm{m}$ (Paul Derbyshire, personal communication). For long cells, the dominant dynamics with $\lambda^{*}=0.5 \mu \mathrm{m}$ are identical to those for $\lambda^{*}=0$, therefore reducing the estimate for $\lambda^{*}$ would not affect the model results. However, for short cells the value of $\lambda^{*}$ does affect the dominant dynamics: the lower estimate, $\lambda^{*}=0.14 \mu \mathrm{m}$, corresponds to $\lambda=0.0070=2.8 \epsilon^{2}$, which would result in the apoplastic flux between the layers not entering the leading-order balance, so that the dynamics would be identical to the $\lambda=0$ case described in Sect. 3.5.2.

In the analysis above, we have neglected any flux between the endodermis and the underlying tissue, whereas Swarup et al. (2005) do not but prescribe an auxin concentration of zero adjacent to the endodermis. A flux into the underlying layers would create a loss term in governing equations $(2.8 \mathrm{a}-\mathrm{c})$, which would lead to corresponding loss terms in the solvability conditions that describe the reduced solutions. In Fig. 8, we present numerical solutions of the discrete cell-based model with both no-flux and 
(a) $t=0.5 \epsilon^{2}$

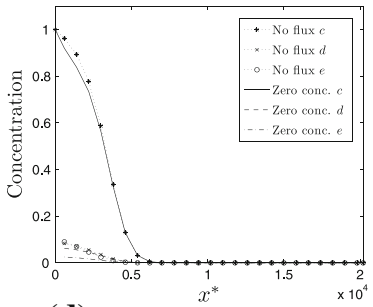

(d) $t=3 \epsilon^{2}$

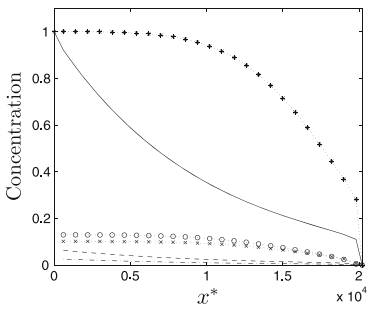

(b) $t=\epsilon^{2}$

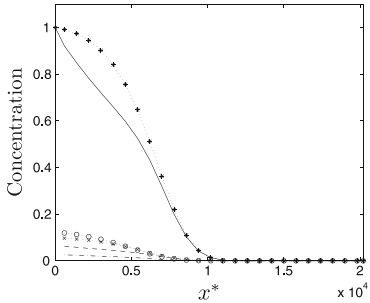

(e) $t=4 \epsilon^{2}$

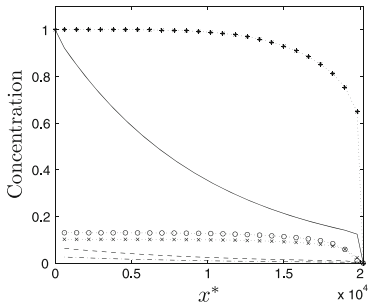

(c) $t=2 \epsilon^{2}$

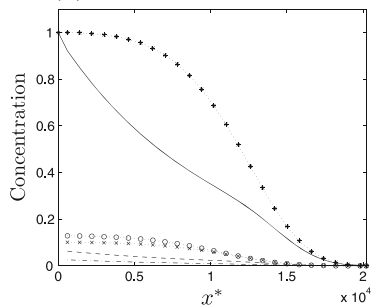

(f) $t=5 \epsilon^{2}$

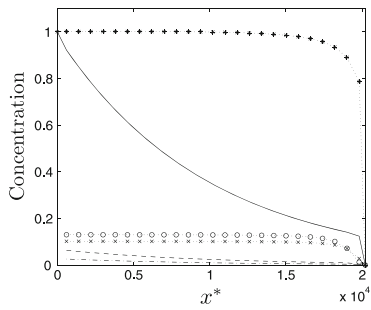

Fig. 8 The influence of the boundary conditions adjacent to the endodermis on the long-cell dynamics. We present solutions of the discrete cell-based model, (3.4), considering both no-flux and zero-concentration boundary conditions and using the long-cell parameter values in Table 4 . We use $\epsilon=0.01$ for illustrative purposes. The solutions with a no-flux boundary condition are identical to those in Fig. 5

zero-concentration boundary conditions: these show that a flux into the underlying layers can create significant gradients through the elongation zone. To distinguish which of these regimes is most appropriate, it would be instructive to analyse a more comprehensive model that includes all the tissue layers.

\section{Conclusions}

In the root, the hormone auxin regulates a range of developmental processes; therefore elucidating the movement of auxin through the tissue should provide important insight into root growth. Many computational cell-scale models are being developed to investigate auxin transport (de Reuille et al. 2006; Feugier and Iwasa 2006; Feugier et al. 2005; Grieneisen et al. 2007; Heisler and Jönsson 2006; Jönsson et al. 2006; Kramer 2004, 2009; Laskowski et al. 2008; Merks et al. 2007; Mironova et al. 2010; Perrine-Walker et al. 2010; Rolland-Lagan and Prusinkiewicz 2005; Smith et al. 2006; Stoma et al. 2008; Swarup et al. 2005); however, little has been done to determine the corresponding tissue-scale descriptions. In this paper, we demonstrate the potential of using (analytical) multiscale methods to simplify an auxin-transport model and to identify the combinations of cell-scale processes that govern the tissue-scale auxin flux. The approach could be extended to include more biological processes, for example, auxin production and degradation, saturable carrier-mediated transport, cell growth, cytoplasmic structure, spatial variations in PIN-carriers' strength and PIN relocation; for simplicity, we have in the above neglected these processes, although they could rather readily be considered by extending the current model. In addition, our analytical 
Table 5 As described in Sect. 4, the dominant tissue-scale behaviour in the wild-type plants and knockout mutants can be described by one of these dimensional governing equations

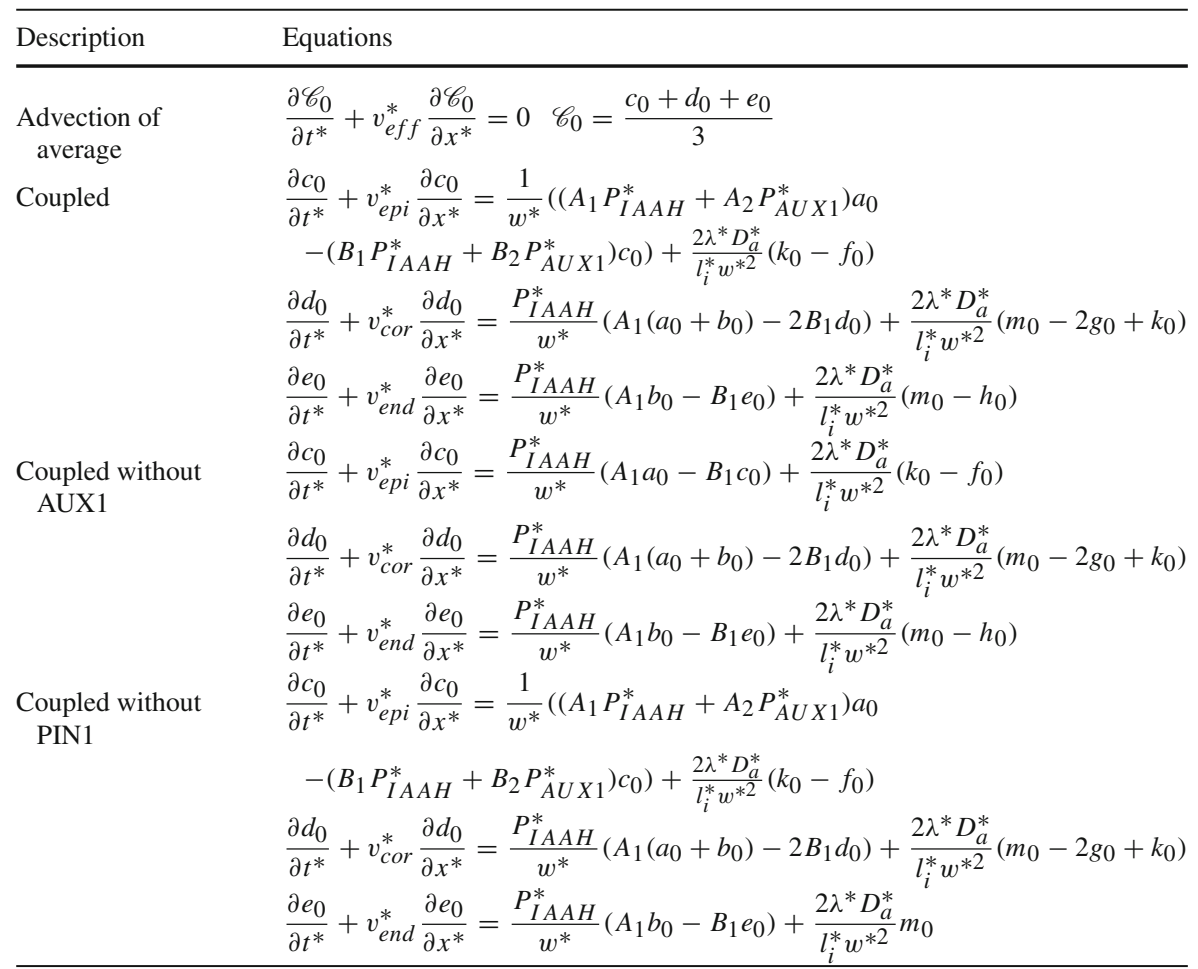

These take the form of either a single advective equation in terms of the average concentration, (3.15), (labelled 'advection of average') or three coupled partial differential equations for the concentrations in each layer, (3.32), (labelled 'coupled', 'coupled without AUX1' and 'coupled without PIN1'). In each case, the governing equations have been redimensionalised using (3.1-3.3)

approach could be applied to more comprehensive root models, for example, to gain further insight into the cell-based model in Grieneisen et al. (2007), where all the tissue layers in the root elongation zone and meristem were considered and auxin-dependent cell division and cell growth were incorporated.

We base our analysis on the elongation-zone model presented by Swarup et al. (2005). Although there are differences between the model analysed here and that by Swarup et al. (2005) (as discussed in Appendix C), there is qualitatively good agreement in our conclusions about the importance of the influx carriers in creating high epidermal concentrations and an overall shootward flux. Our analysis provides additional insight into the relative importance of the different flux components. Our key finding is that the tissue-scale dynamics can be captured by a number of distinguished limits and these have enabled us to identify several systems of partial differential equations that describe the overall dynamics in the different biological cases; the dimensional versions of these equations are summarised in Table 5. In each case, relatively simple expressions are available for the key tissue-scale parameters (for example, the effective 
Table 6 Summary of the dominant tissue-scale behaviour and effective dimensional parameters in the wild-type plants and knockout mutants

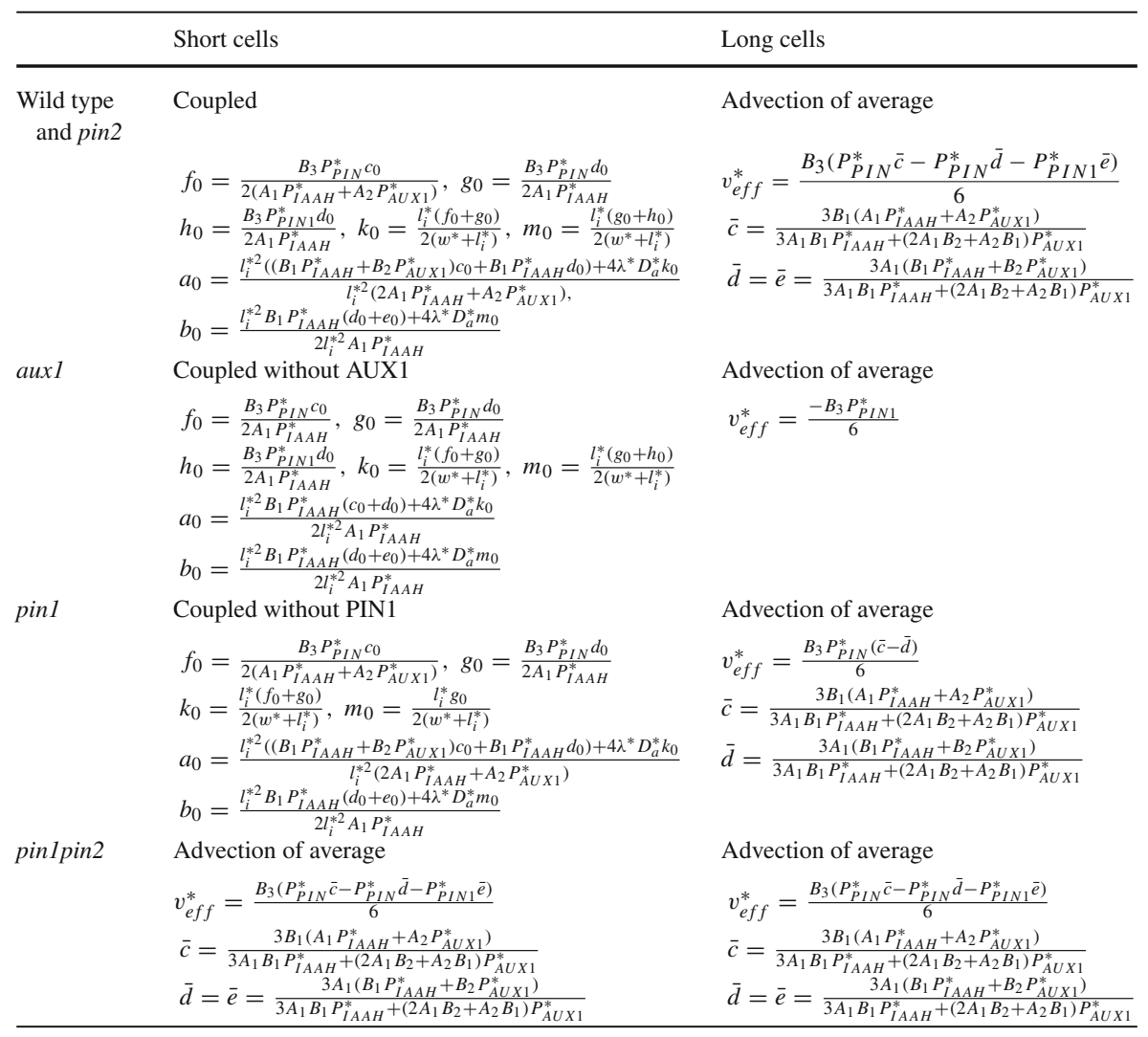

The governing equations are referred to using the descriptions detailed in Table 5 . Where the dynamics are described by coupled equations, dimensional versions of $(3.14 \mathrm{~d}-\mathrm{j})$ determine the relationship between the cytoplasmic and apoplastic concentrations. In the other cases, we have a single equation for the average concentration, with the effective velocity given by (3.28) and the proportionality constants being solutions of (3.14a-h) (all after appropriate rescaling and simplification)

velocities associated with directed transport), and the resulting formulae clearly show how the cell and apoplast dimensions and the membrane transport components affect the tissue-scale flux (see Table 6). In particular, we find the overall behaviour depends on the cell length: for short cells close to the root tip, auxin spreads between the three tissue layers on the same time scale as it propagates the length of the tissue, whereas for the longer cells, auxin distributes between the layers rapidly and maintains equal proportions as the wave propagates.

One of our focusses is the interplay between transport across cell membranes and diffusion within the apoplast. Although one might expect the diffusive flux through the apoplast to contribute on the tissue scale, our analysis suggests that these dynamics are not biologically relevant for the parameter estimates in the current literature. However, on the basis of the distinguished limits that include this apoplastic flux presented here, one can easily assess their applicability should parameter estimates change, or 
be different for another plant species. Although the tissue-scale apoplastic flux is negligible, we find that, with an apoplast thickness of around $0.5 \mu \mathrm{m}$, apoplastic diffusion between nearby cells affects the auxin distribution between the three layers for short cells, suggesting that including apoplastic diffusion in auxin-transport models can be important; however, we emphasise that this conclusion depends on the cell length, apoplastic thickness and PIN permeabilities, and the methods presented here should be instructive in assessing whether to include apoplastic diffusion in future models.

The analysis provides insight into several agravitropic Arabidopsis thaliana mutants. In auxl mutants, the lack of influx carriers in the epidermal layer results in the effective tissue-scale auxin velocity being in the rootward (i.e. reverse) direction. A gravitropic response requires shootward auxin transport through the root's outer layers to transmit the signal, sensed in the columella cells at the root tip, to the elongating cells (Swarup et al. 2005). Therefore the lack of shootward flux in the auxl mutants leads to their agravitropic phenotype. In addition, gravitropism requires the shootward flux to be sufficiently rapid: should the shootward flux be slow, azimuthal variations in the auxin concentration at the root tip will be smoothed out by azimuthal diffusion before the auxin reaches the elongating tissue, resulting in an agravitropic plant root. We predict that this smoothing out of azimuthal variations will occur in the agravitropic pin 2 single mutants and pin1pin2 double mutants, for which shootward auxin transport is much slower than in wild-type roots. In addition, we showed that the location of the cortical cell's PIN carriers significantly affects the amount of auxin that enters the elongation zone, which may also have implications for the regulation of the gravitropic response.

Acknowledgments This work was carried out at the Centre for Plant Integrative Biology (CPIB), and we are grateful to both the Biotechnology and Biological Sciences Research Council (BBSRC) and the Engineering and Physical Sciences Research Council (EPSRC) for supporting this project financially. In addition, JRK gratefully acknowledges the support of the Royal Society and Wolfson Foundation. We would like to thank our colleagues and collaborators at CPIB, in particular, Prof. Malcolm Bennett, Dr Eric Kramer, Dr Ranjan Swarup and Prof. Tobias Baskin.

Open Access This article is distributed under the terms of the Creative Commons Attribution Noncommercial License which permits any noncommercial use, distribution, and reproduction in any medium, provided the original author(s) and source are credited.

\section{Appendix A: Derivation of the carrier-mediated fluxes}

In this section, we derive the carrier-mediated flux terms (2.3). According to Goldman-Hodgkin-Katz theory, the carrier-mediated flux has two components-a passive diffusive component and a component driven by the electrochemical gradient across the cell membrane-and is described by the Nerst-Planck equation:

$$
J^{*}=-D_{m}^{*}\left(\frac{\partial c^{*}}{\partial x_{m}^{*}}+\frac{z F_{D} c^{*}}{R T^{*}} \frac{\partial \phi^{*}}{\partial x_{m}^{*}}\right),
$$

where $J^{*}$ is the flux per unit length through the membrane, $c^{*}$ is the concentration within the membrane, $x_{m}^{*}$ is the distance through the membrane $\left(x_{m}^{*}=0\right.$ denoting 
the boundary of the cytoplasm and the membrane), $D_{m}^{*}$ is the coefficient of diffusion within the membrane, $\phi^{*}$ is the potential within the membrane, $z$ is the valence of the molecule, $F_{D}$ is Faraday's constant, $R$ is the gas constant and $T^{*}$ is the temperature (which we take to be constant). We assume the electric field to be uniform within the membrane, i.e. $\partial \phi^{*} / \partial x_{m}=-V^{*} / l_{m}^{*}$, where $V^{*}$ is the potential difference across the membrane (i.e. the cytoplasmic potential minus apoplastic potential) and $l_{m}^{*}$ is the membrane thickness. Then, supposing that the timescale of transport across the membrane is sufficiently fast that the flux, $J^{*}$, can be treated as uniform within it, we can solve (5.1) to find

$$
J^{*}=\frac{D_{m}^{*}}{l_{m}^{*}}\left(\left.q(-z \tilde{\phi}) c^{*}\right|_{x_{m}^{*}=0}-\left.q(z \tilde{\phi}) c^{*}\right|_{x_{m}^{*}=l_{m}^{*}}\right),
$$

where $q(x)=x /\left(e^{x}-1\right)$ and $\tilde{\phi} \equiv F_{D} V^{*} / R T^{*}$.

The PIN efflux carriers transport single molecules of anionic auxin which have a valence $z=-1$. Considering the cell membrane face labelled M on Fig. 2, for example, the PIN-mediated flux from the cytoplasm $\mathrm{C}$ into the apoplast region $\mathrm{F}$ is given by (5.2) with $\left.c^{*}\right|_{x_{m}^{*}=0}=\left(1-B_{1}\right) c_{i}^{*}$ and $\left.c^{*}\right|_{x_{m}^{*}=l_{m}^{*}}=\left(1-A_{1}\right) f_{i}^{*}$, which yields

$$
J_{P I N}^{*}=P_{P I N}^{*}\left(q(\tilde{\phi})\left(1-B_{1}\right) c_{i}^{*}-q(-\tilde{\phi})\left(1-A_{1}\right) f_{i}^{*}\right)
$$

where $P_{P I N}^{*}=D_{m}^{*} / l_{m}^{*}$ is the effective membrane permeability due to the PIN carriers and reflects both the efflux-carrier density and strength. In contrast, the AUX1 influx carriers cotransport two protons with each anion of auxin (Lomax et al. 1985); this complex has a positive valence, $z=1$, and will travel down the potential gradient from the apoplast into the cytoplasm. Therefore, from (5.2), the AUX1 carriers mediate a flux from cytoplasm $\mathrm{C}$ to apoplast region $\mathrm{F}$ of the form

$$
J_{A U X 1}^{*}=-P_{A U X 1}^{*}\left(q(\tilde{\phi})\left(1-A_{1}\right) f_{i}^{*}-q(-\tilde{\phi})\left(1-B_{1}\right) c_{i}^{*}\right),
$$

where $P_{A U X 1}^{*}=D_{m}^{*} / l_{m}^{*}$ is the effective permeability due to the influx carriers (and we note that $D_{m}^{*}$ may be different for the different types of carrier).

\section{Appendix B: Biological parameter estimates}

We now discuss our choice of appropriate parameter estimates. We take the root elongation zone to consist of layers of $N \approx 20$ cells that have equal width $w^{*}=10 \mu \mathrm{m}$ and that increase in length through the elongation zone by approximately 20 -fold, from $10 \mu \mathrm{m}$ to $200 \mu \mathrm{m}$ (Swarup et al. 2007). Although the cell number and length varies slightly between the different cell types (Swarup et al. 2007), we neglect these difference for simplicity. The dominant dynamics depend on the cytoplasm length, and therefore in the analysis that follows, we consider separately short cells and long cells, with representative cytoplasm lengths of $l_{T}^{*}=20 \mu \mathrm{m}$, and $l_{T}^{*}=200 \mu \mathrm{m}$ respectively (these cases lead to distinct distinguished limits). Swarup et al. (2005) use an apoplast 
thickness of $\lambda^{*}=0.5 \mu \mathrm{m}$ throughout the elongation zone (assuming that deposition of new material maintains the cell-wall thickness during elongation). However, recent measurements suggest a lower value of $0.14 \mu \mathrm{m}$ (Paul Derbyshire, personal communication); we therefore use the model to explore how the apoplast thickness affects the tissue-scale dynamics.

The cell-membrane permeabilities, $P_{I A A H}^{*}, P_{A U X 1}^{*}, P_{P I N}^{*}$, and $P_{P I N 1}^{*}$, are key model parameters. Delbarre et al. $(1996,1994)$ measured the passive-diffusion membrane permeability in tobacco cells as $0.14-0.18 \mathrm{~cm} \mathrm{~h}^{-1}$ and the majority of previous modelling studies use estimates around these values (Chavarria-Krauser et al. 2005; Goldsmith et al. 1981; Heisler and Jönsson 2006; Jönsson et al. 2006; Kramer 2004, 2009; Swarup et al. 2005); thus following Swarup et al. (2005), we set $P_{I A A H}=0.2 \mathrm{~cm} \mathrm{~h}^{-1}=0.56 \mu \mathrm{m} \mathrm{s}^{-1}$. Experimental values for the membrane permeabilities due to the influx and efflux carriers have not been well characterised. Delbarre et al. (1996) reports a carrier-mediated auxin influx and efflux in suspensioncultured tobacco cells, which has been used to estimate an influx carrier permeability of $0.02 \mathrm{~cm} \mathrm{~h}^{-1}$ and an efflux carrier permeability of $0.01 \mathrm{~cm} \mathrm{~h}^{-1}$ (Swarup et al. 2005). In addition, Szponarski et al. (1999) measured the influx carrier permeability to be $0.011 \mathrm{~cm} \mathrm{~h}^{-1}$ in plasma-membrane vesicles derived from mature Arabidopsis thaliana leaves. However, these estimates are an average over all the cells therefore we would expect the actual values to be larger for the cell membranes containing AUX1 and PIN proteins. The majority of previous models use the same value for the permeability due to the influx carriers, and therefore we set $P_{A U X 1}=0.2 \mathrm{~cm} \mathrm{~h}^{-1}=0.56 \mu \mathrm{m} \mathrm{s}^{-1}$ (Heisler and Jönsson 2006; Kramer 2004; Swarup et al. 2005). In contrast, various values are used for the permeability due to the efflux carriers, including $0.124 \mu \mathrm{m} \mathrm{s}^{-1}$ (Goldsmith et al. 1981; Jönsson et al. 2006), $0.27 \mu \mathrm{m} \mathrm{s}^{-1}$ (Heisler and Jönsson 2006), $1.4 \mu \mathrm{m} \mathrm{s}^{-1}$ (Kramer 2004) and $20 \mu \mathrm{m} \mathrm{s}^{-1}$ (Laskowski et al. 2008). Swarup et al. (2005) assume that all the epidermal cells (and similarly all the cortical cells and all the endodermal cells) have an efflux-carrier number proportional to the area of the cell membrane, which, as the efflux carriers are present only on the shootward face of the membrane, results in the efflux-carrier density on this face increasing with cell length. As far as we are aware, there is no conclusive evidence that the shootward face's efflux-carrier density varies with cell length, therefore we study here the simpler case in which the efflux-carrier density, and hence the resulting membrane permeability, is independent of cell length. We use representative values of $P_{P I N}^{*}=0.56 \mu \mathrm{m} \mathrm{s}^{-1}$ and $P_{P I N 1}^{*}=0.28 \mu \mathrm{m} \mathrm{s}^{-1}$ which correspond to the PIN1 and PIN2 carriers having equal strength and the total PIN carrier density for membranes containing both PIN1 and PIN2 being double that for membranes with PIN1 alone. We use the model to investigate how the permeabilities chosen affect the tissue-scale dynamics.

Previous models assume that auxin diffuses within the cytoplasm as it does in water, with a diffusion coefficient of $D_{c}^{*}=6.7 \times 10^{-10} \mathrm{~m}^{2} \mathrm{~s}^{-1}$, whereas the cell-wall constituents reduce the diffusion rate in the apoplast (Heisler and Jönsson 2006; Kramer 2006, 2009; Swarup et al. 2005). Recent experimental work (Kramer et al. 2007) has confirmed apoplastic diffusion to be slower than that in the cytoplasm, and found that $D_{a}^{*}=3.2 \times 10^{-11} \mathrm{~m}^{2} \mathrm{~s}^{-1}$. The remaining parameter values, $p H_{c}, p H_{w}, p K$ and $V^{*}$, are well characterised, and we use the representative values given in Swarup et al. (2005); these parameters are used to calculate the constants $(2.1,2.4)$. 


\section{Appendix C: Comparison with Swarup et al. (2005)}

The auxin-transport model analysed in this paper is based on the cell-scale model of Swarup et al. (2005), who studied auxin transport in the root elongation zone by considering a multi-layered 3D cylinder of cells. As noted in Sects. 2.1 and 2.2, the key differences in the model assumptions used here are that (I) we consider a two-dimensional configuration; (II) we assume that for each cell type, the efflux permeability is equal for each cell, whereas in Swarup et al. (2005) these permeabilities depend on cell length; and (III) Swarup et al. (2005) discretise the cytoplasmic compartments to capture non-homogeneous cytoplasmic concentrations and model the cytoplasmic diffusion, a process that we can take to be rapid for the efflux permeabilities used here (see Sect. 2.2). We remark that we adopt at the start the 'simplest' assumptions, for clarity of analysis and to aid the identification of the key lumped parameters that dominate the dynamics on the tissue scale, the goal here being to maximise intuition into such matters rather than address all the relevant complications. We also consider different boundary conditions: Swarup et al. (2005) include the flux into the underlying tissue by prescribing an auxin concentration of zero adjacent to the endodermis; in contrast, we assume no flux from the endodermis into the underlying tissue to maintain analytical tractability. We discuss how these boundary conditions affect the dynamics in Sect. 4.3.

\section{References}

Benjamins R, Scheres B (2008) Auxin: the looping star in plant development. Annu Rev Plant Biol 59:443465

Benková E, Michniewicz M, Sauer M, Teichmann T, Seifertová D, Jürgens G, Friml J (2003) Local, effluxdependent auxin gradients as a common module for plant organ formation. Cell 115:591-602

Berleth T, Scarpella E, Prusinkiewicz P (2007) Towards the systems biology of auxin-transport-mediated patterning. Trends Plant Sci 12:151-159

Blilou I, Xu J, Wildwater M, Willemsen V, Paponov I, Friml J, Heidstra R, Aida M, Palme K, Scheres B (2005) The PIN auxin efflux facilitator network controls growth and patterning in Arabidopsis roots. Nature 433:39-44

Casimiro I, Marchant A, Bhalerao R, Beeckman T, Dhooge S, Swarup R, Graham N, Inzé D, Sandberg G, Casero P, Bennett MJ (2001) Auxin transport promotes Arabidopsis lateral root initiation. Plant Cell 13:843-852

Casimiro I, Beeckman T, Graham N, Bhalerao R, Zhang H, Casero P, Sandberg G, Bennett MJ (2003) Dissecting Arabidopsis lateral root development. Trends Plant Sci 8(4):165-171

Chavarría-Krauser A, Ptashnyk M (2009) Homogenization of long-range auxin transport in plant tissues. Nonlinear Anal Real. doi:101016/jnonrwa200812003

Chavarria-Krauser A, Jager W, Schurr U (2005) Primary root growth: a biophysical model of auxin-related control. Funct Plant Biol 32:849-862

de Reuille PB, Bohn-Courseau I, Ljung K, Morin H, Carraro N, Godin C, Traas J (2006) Computer simulations reveal properties of the cell-cell signaling network at the shoot apex in Arabidopsis. Proc Natl Acad Sci USA 103:1627-1632

De Smet I, Tetsumura T, De Rybel B, Frey NF, Laplaze L, Casimiro I, Swarup R, Naudts M, Vanneste S, Audenaert D et al (2007) Auxin-dependent regulation of lateral root positioning in the basal meristem of Arabidopsis. Development 134:681-690

Delbarre A, Muller P, Imhoff V, Morgat JL, Barbier-Brygoo H (1994) Uptake, accumulation and metabolism of auxins in tobacco leaf protoplasts. Planta 195:159-167 
Delbarre A, Muller P, Imhoff V, Guern J (1996) Comparison of mechanisms controlling uptake and accumulation of 2, 4-dichlorophenoxy acetic acid, naphthalene-1-acetic acid, and indole-3-acetic acid in suspension-cultured tobacco cells. Planta 198:532-541

Feugier FG, Iwasa Y (2006) How canalization can make loops: a new model of reticulated leaf vascular pattern formation. J Theor Biol 243:235-244

Feugier FG, Mochizuki A, Iwasa Y (2005) Self-organization of the vascular system in plant leaves: interdependent dynamics of auxin flux and carrier proteins. J Theor Biol 236:366-375

Friml J (2003) Auxin transport-shaping the plant. Curr Opin Plant Biol 6:7-12

Friml J, Wisniewska J, Benkova E, Mendgen K, Palme K (2002) Lateral relocation of auxin efflux regulator PIN3 mediates tropism in Arabidopsis. Nature 415:806-809

Goel P, Sneyd J, Friedman A (2006) Homogenization of the cell cytoplasm: the calcium bidomain equations. Multiscale Model Simul 5:1045-1062

Goldsmith MHM, Goldsmith TH, Martin MH (1981) Mathematical analysis of the chemosmotic polar diffusion of auxin through plant tissues. Proc Natl Acad Sci USA 78:976-980

Grieneisen VA, Xu J, Marée AFM, Hogeweg P, Scheres B (2007) Auxin transport is sufficient to generate a maximum and gradient guiding root growth. Nature 449:1008-1013

Heisler M, Jönsson H (2006) Modeling auxin transport and plant development. J Plant Growth Regul 25:302-312

Jiang K, Feldman LJ (2005) Regulation of root apical meristem development. Annu Rev Cell Dev Biol 21:485-509

Jönsson H, Krupinski P (2010) Modeling plant growth and pattern formation. Curr Opin Plant Biol 13:5-11

Jönsson H, Heisler MG, Shapiro BE, Meyerowitz EM, Mjolsness E (2006) An auxin-driven polarized transport model for phyllotaxis. Proc Natl Acad Sci USA 103:1633-1638

Keener J, Sneyd J (2004) Mathematical physiology. Springer, USA

Kramer EM (2002) A mathematical model of pattern formation in the vascular cambium of trees. J Theor Biol 216:147-158

Kramer EM (2004) PIN and AUX/LAX proteins: their role in auxin accumulation. Trends Plant Sci 9:578582

Kramer EM (2006) How far can a molecule of weak acid travel in the apoplast or xylem? Plant Physiol 141:1233-1236

Kramer EM (2008) Computer models of auxin transport: a review and commentary. J Exp Bot 59:45-53

Kramer EM (2009) Auxin-regulated cell polarity: an inside job? Trends Plant Sci 14:242-247

Kramer EM, Bennett MJ (2006) Auxin transport: a field in flux. Trends Plant Sci 11:382-386

Kramer EM, Frazer NL, Baskin TI (2007) Measurement of diffusion within the cell wall in living roots of Arabidopsis thealiana. J Exp Bot 58:3005-3015

Kramer EM, Draye X, Bennett MJ (2008) Practical systems biology, chap Modelling root growth and development. Taylor and Francis group, New York, USA

Krupinski P, Jönsson H (2010) Modeling auxin-regulated development. Cold Spring Harb Perspect Biol 2:a001,560

Laskowski M, Grieneisen VA, Hofhuis H, ten Hove CA, Hogeweg P, Marée AFM, Scheres B (2008) Root system architecture from coupling cell shape to auxin transport. PLoS Biol 6:e307

Lomax TL, Mehlhorn RJ, Briggs WR (1985) Active auxin uptake by zucchini membrane vesicles: quantitation using ESR volume and $\Delta \mathrm{pH}$ determinations. Proc Natl Acad Sci USA 82:6541-6545

Marchant A, Kargul J, May ST, Muller P, Delbarre A, Perrot-Rechenmann C, Bennett MJ (1999) AUX1 regulates root gravitropism in Arabidopsis by facilitating auxin uptake within root apical tissues. EMBO J 18:2066-2073

Martin MH, Goldsmith MHM, Goldsmith TH (1990) On polar auxin transport in plant cells. J Math Biol 28:197-223

Merks RMH, Vande Peer Y, Inzé D, Beemster GTS (2007) Canalization without flux sensors: a travelingwave hypothesis. Trends Plant Sci 12:384-390

Mironova VV, Omelyanchuk NA, Yosiphon G, Fadeev SI, Kolchanov NA, Mjolsness E, Likhoshvai VA (2010) A plausible mechanism for auxin patterning along the developing root. BMC Syst Biol 4:98

Mitchison GJ (1980) A model for vein formation in higher plants. Proc R Soc Lond B Biol 207:79-109

Mitchison GJ (1980) The dynamics of auxin transport. Proc R Soc Lond B Biol 209:489-511

Mitchison GJ, Hanke DE, Sheldrake AR (1981) The polar transport of auxin and vein patterns in plants. Philos Trans R Soc B 295:461-471 
Newell AC, Shipman PD, Sun Z (2008) Phyllotaxis: cooperation and competition between mechanical and biochemical processes. J Theor Biol 251:421-439

Ottenschlager I, Wolff P, Wolverton C, Bhalerao RP, Sandberg G, Ishikawa H, Evans M, Palme K (2003) Gravity-regulated differential auxin transport from columella to lateral root cap cells. Proc Natl Acad Sci USA 100:2987-2991

Peer WA, Bandyopadhyay A, Blakeslee JJ, Makam SN, Chen RJ, Masson PH, Murphy AS (2004) Variation in expression and protein localization of the PIN family of auxin efflux facilitator proteins in flavonoid mutants with altered auxin transport in Arabidopsis thaliana. Plant Cell 16:1898-1911

Péret B, De Rybel B, Casimiro I, Benková E, Swarup R, Laplaze L, Beeckman T, Bennett MJ (2009) Arabidopsis lateral root development: an emerging story. Trends Plant Sci 14:399-408

Perrine-Walker F, Doumas P, Lucas M, Vaissayre V, Beauchemin NJ, Band LR, Chopard J, Crabos A, Conejero G, Péret B, King JR, Verdeil JL, Hocher V, Franche C, Bennett MJ, Tisa LS, Laplaze L (2010) Auxin carriers localization drives auxin accumulation in plant cells infected by Frankia in Casuarina glauca actinorhizal nodules. Plant Physiol 154:1372-1380

Rahman A, Takahashi M, Shibasaki K, Wu S, Inaba T, Tsurumi S, Baskin TI (2010) Gravitropism of Arabidopsis thaliana roots requires the polarization of PIN2 toward the root tip in meristematic cortical cells. Plant Cell 22:1762-1776

Rashotte AM, Brady SR, Reed RC, Ante SJ, Muday GK (2000) Basipetal auxin transport is required for gravitropism in roots of Arabidopsis. Plant Physiol 122:481-490

Rolland-Lagan AG, Prusinkiewicz P (2005) Reviewing models of auxin canalization in the context of leaf vein pattern formation in Arabidopsis. Plant J 44:854-865

Shipley RJ, Jones GW, Dyson RJ, Sengers BG, Bailey CL, Catt CJ, Please CP, Malda J (2009) Design criteria for a printed tissue engineering construct: a mathematical homogenization approach. $\mathrm{J}$ Theor Biol 259:489-502

Smith RS, Bayer EM (2009) Auxin transport-feedback models of patterning in plants. Plant Cell Environ 32:1258-1271

Smith RS, Guyomarc'h S, Mandel T, Reinhardt D, Kuhlemeier C, Prusinkiewicz P (2006) A plausible model of phyllotaxis. Proc Natl Acad Sci USA 103:1301-1306

Stoma S, Lucas M, Chopard J, Schaedel M, Traas J, Godin C (2008) Flux-based transport enhancement as a plausible unifying mechanism for auxin transport in meristem development. PLoS Comput Biol 4:e1000,207

Swarup R, Kramer EM, Perry P, Knox K, Leyser HMO, Haseloff J, Beemster GTS, Bhalerao R, Bennett MJ (2005) Root gravitropism requires lateral root cap and epidermal cells for transport and response to a mobile auxin signal. Nat Cell Biol 7:1057-1065

Swarup R, Perry P, Hagenbeek D, Van Der Straeten D, Beemster GTS, Sandberg G, Bhalerao R, Ljung K, Bennett MJ (2007) Ethylene upregulates auxin biosynthesis in Arabidopsis seedlings to enhance inhibition of root cell elongation. Plant Cell 19:2186-2196

Szponarski W, Guibal O, Espuna M, Doumas P, Rossignol M, Gibrat R (1999) Reconstitution of an electrogenic auxin transport activity mediated by Arabidopsis thaliana plasma membrane proteins. FEBS lett 446:153-156

Tanaka H, Dhonukshe P, Brewer PB, Friml J (2006) Spatiotemporal asymmetric auxin distribution: a means to coordinate plant development. Cell Mol Life Sci 63:2738-2754

Twycross J, Band LR, Bennett MJ, King JR, Krasnogor N (2010) Stochastic and deterministic multiscale models for systems biology: an auxin-transport case study. BMC Syst Biol 4:34

Ubeda-Tomás S, Federici F, Casimiro I, Beemster GTS, Bhalerao R, Swarup R, Doerner P, Haseloff J, Bennett MJ (2009) Gibberellin signaling in the endodermis controls Arabidopsis root meristem size. Curr Biol 19:1194-1199

Vieten A, Sauer M, Brewer PB, Friml J (2007) Molecular and cellular aspects of auxin-transport-mediated development. Trends Plant Sci 12:160-168 